\title{
Adoption of Blockchain Technology through Digital Twins in the Construction Industry 4.0: A PESTELS Approach
}

\author{
Benjamin Teisserenc* ${ }^{(D)}$ and Samad Sepasgozar (D) \\ Faculty of Built Environment, The University of New South Wales, Sydney, NSW 2052, Australia; \\ Sepas@unsw.edu.au \\ * Correspondence: b.teisserenc@unsw.edu.au
}

Citation: Teisserenc, B.; Sepasgozar,

S. Adoption of Blockchain

Technology through Digital Twins in the Construction Industry 4.0: A PESTELS Approach. Buildings 2021, 11,670. https://doi.org/10.3390/ buildings 11120670

Academic Editors: Zoubeir Lafhaj and Zakaria Dakhli

Received: 29 October 2021

Accepted: 13 December 2021

Published: 20 December 2021

Publisher's Note: MDPI stays neutral with regard to jurisdictional claims in published maps and institutional affiliations.

Copyright: (c) 2021 by the authors. Licensee MDPI, Basel, Switzerland. This article is an open access article distributed under the terms and conditions of the Creative Commons Attribution (CC BY) license (https:/ / creativecommons.org/licenses/by/ $4.0 /)$.

\begin{abstract}
The key challenges of the building, engineering, construction, operations, and mining (BECOM) industries are the lack of trust, inefficiencies, and the fragmentation of the information value chain into vulnerable data silos throughout the lifecycle of projects. This paper aims to develop a novel conceptual model for the implementation of blockchain technology (BCT) for digital twin(s) (DT) in the BECOM industry 4.0 to improve trust, cyber security, efficiencies, information management, information sharing, and sustainability. A PESTELS approach is used to review the literature and identify the key challenges affecting BCT adoption for the BECOM industry 4.0. A review of the technical literature on BCT combined with the findings from PESTELS analysis permitted researchers to identify the key technological factors affecting BCT adoption in the industry. This allowed offering a technological framework - namely, the decentralized digital twin cycle (DDTC) — that leverages BCT to address the key technological factors and to ultimately enhance trust, security, decentralization, efficiency, traceability, and transparency of information throughout projects' lifecycles. The study also identifies the gaps in the integration of BCT with key technologies of industry 4.0, including the internet of things (IoT), building information modeling (BIM), and DT. The framework offered addresses key technological factors and narrows key gaps around network governance, scalability, decentralization, interoperability, energy efficiency, computational requirements, and BCT integration with IoT, BIM, and DT throughout projects' lifecycles. The model also considers the regulatory aspect and the environmental aspect, and the circular economy (CE). The theoretical framework provides key technological building blocks for industry practitioners to develop the DDTC concept further and implement it through experimental works. Finally, the paper provides an industry-specific analysis and technological approach facilitating BCT adoption through DT to address the key challenges and improve sustainability for the BECOM industry 4.0.
\end{abstract}

Keywords: blockchain; PESTELS; construction; built environment; mining; BIM; IoT; digital twin; industry 4.0

\section{Introduction}

\subsection{General Information}

\subsubsection{The BECOM Industry}

The BECOM Industry, as one of the largest industries in the world, faces key challenges such as decreasing productivity over the last decades compared to other manufacturing industries [1]. The BECOM industry is fragmented [2] as organizations work in silos in an adversarial manner [3], and the contractors act as central parties [4]. There are inefficiencies in the construction supply chain and rework due to miscommunication and inaccurate or inaccessible information, and this cost around \$31 billion in the US construction industry in 2018 [5]. Lack of trust is a major challenge [6] for the BECOM industry; poor regulations and compliance are also key issues in the industry [7]. The BECOM industry is slow to adopt new technologies [7] compared to other manufacturing industries. The industry's slower digitization [1] is due to short-term thinking, uncertainty, fragmentation of the 
supply chain, lack of R\&D investments, and a rigid culture regarding organizational and process changes [2]. The short duration and unique complexity of construction projects [2] lead to short-term thinking and hesitation to change. Short-term thinking is, however, less critical in the mining industry, where projects can last for decades.

\subsubsection{Industry 4.0 Technologies}

The fourth industrial revolution governed by digital technologies and the digitization of processes is referred to as industry 4.0. The key technologies of the BECOM industry 4.0 are BIM, cloud computing, mobile computing, mixed reality (MR), cyber-physical systems (CPS), robotics, IoT, and Big Data [1]. IoT can save from $\$ 160$ billion to $\$ 930$ billion each year on construction sites and mines [8]. However, the management of IoT networks is centralized [9] and hence prone to a single point of failure. BIM Level 1 and Level 2 are also centralized and prone to generate data silos [10], while BIM Level 3 is more collaborative but still relies on centralized clouds.

$\mathrm{BCT}$ is decentralized and secure by nature, and it has the potential to decentralize IoT networks governance and BIM management and improve security, traceability, and data integrity. BCT emerged in 2008 with the creation of the Bitcoin protocol [11] that uses open-source code. BCT has already shown its capacity to offer a decentralized, secure, transparent, open, auditable, and immutable shared ledger of transactions for the financial and supply chain industries. BCT should generate more than USD $\$ 175$ billion by 2025 and over USD $\$ 3$ trillion by 2030 [12].

The disruptive potential of BCT in the built environment was imagined with the "Blockchain of Circular BIM things" [13]. The large benefits of blockchain and its impact on construction businesses can justify its adoption. Since BCT has a significant role in connecting different technologies, devices, and stakeholders through peer-to-peer networks, its adoption will affect the connectivity [14] as a core promise for the BECOM industry 4.0. Leveraging BCT for the BECOM supply chain can decrease fragmentation; increase collaboration; improve efficiency; and enhance trust and transparency for processes, information sharing, and information management [15]. BCT is promising for the BECOM industry 4.0 and could contribute to saving costs and time, improving quality, enhancing safety, facilitating on-time and on-budget delivery, and improving communication and customer relationships while strengthening the industry's image [7]. The Australian building confidence report considers that BCT could provide a verifiable shared digital audit trail of regulatory transactions [16].

The integration of BCT for DT, in particular, has great potential for the BECOM industry. DT represents the digital replica of a smart physical asset like a smart building equipped with IoT sensors. DT can generate considerable value throughout projects' lifecycle for processes approval, automated monitoring, as-designed and as-built info, resources planning, logistics, safety monitoring, predictive maintenance, quality assurance, and compliance [17]. DT captures data from the information value chain of projects to improve the performance of assets and assist customers, owners, operators, governments, investors, and society in general [18]. The global market for DT was about USD $\$ 3.2$ billion in 2018 and has a projected value of $\$ 29.1$ billion by 2025 [17]. Greater data sharing could bring $£ 7$ billion per year across the UK infrastructure sectors [19].

The Gemini Principles provide an information management framework for the development of DTs in the built environment [19]. The Gemini Principles specify that the information value chain should be managed to decrease the data volume while the data value is increased [19]. Moreover, the trustworthiness of the data, its security, openness, and quality are key pillars of The Gemini Principles and concurrently the key features of BCT. Hence, BCT could be a suitable technology for DT to satisfy The Gemini Principles. DT ecosystems for the public good should be secure, reliable, trusted, open, transparent, collaborative, with data interoperability (format agnostic) and access control rules guaranteeing privacy [17]. BCT could guarantee the trust, security, and transparency required for integrated shared DT platforms [20] in accordance with The Gemini Principles. 
$\mathrm{BCT}$ can enhance proof of provenance, data trustworthiness, data security, and data traceability for DT of manufacturing supply chains [21]. However, the literature available about BCT for DT is scarce, and some technical gaps need to be addressed, such as the evaluation of technical and non-functional requirements for BCT integration with DT applications, the integration of BCT with BIM and IoT, and the authentication of data to guarantee data integrity. The literature proposes that if distributed ledger technologies such as BCT are developed further and become fully mature, they can become reliable solutions to key challenges such as low productivity and poor financial processes [7].

\subsection{Research Questions}

Since BCT has a strong potential to improve the BECOM industry 4.0, it is important to efficiently facilitate its adoption. Hence it is key to identify what are the barriers affecting BCT adoption in the industry. Secondly, as BCT is a key technology to strengthen data integrity and the trustworthiness of DT, it is essential to evaluate what are the key technological factors affecting the adoption of BCT through DT in the BECOM industry 4.0. Thirdly, as these technologies are emerging and the literature is limited, it is crucial to identify the gaps around the adoption of BCT for DT. Hence the three research questions of this study are as follows:

1. What are the key challenges affecting BCT adoption in the BECOM industry?

2. What are the key technological factors affecting BCT adoption with DT in the industry?

3. What are the technological gaps around the adoption of BCT for DT in the industry?

\subsection{Aim and Scope}

The aim of this paper is to develop a technological framework, based on a novel conceptual model, for the integration of BCT with DT for projects of the BECOM industry 4.0. The conceptual model aspires to address the key challenges of the BECOM industry, and the aimed technological framework will contribute to narrowing the technical gaps around the combination of these technologies. Hence, to answer the research questions, the objectives of the paper are as follows.

1. The first objective is to identify the challenges of the BECOM industry 4.0 that either affect or can be improved with BCT adoption.

2. The second objective is to evaluate the key technological factors affecting the adoption of BCT in the BECOM industry 4.0.

3. The third objective is to propose a conceptual model for the integration of BCT and DT for projects of the BECOM industry 4.0.

For this purpose, this study reviews the literature about BCT and DT in the context of the BECOM industry 4.0. The first contribution of this paper is in Section 3, which presents the literature review following a PESTELS approach [2] to identify the key challenges affecting BCT adoption in relation to each PESTELS theme. The second contribution is in Section 4, which identifies the key technological factors affecting the adoption of BCT in the BECOM industry 4.0. The paper's main contribution is presented in Section 5 with the novel technological framework based on a conceptual model called the decentralized digital twin cycle (DDTC). The fourth contribution of the study is the identification of technological gaps concerning the adoption of BCT for DTs in BECOM 4.0. Finally, the paper discusses in Section 6 the contributions of the offered DDTC technological framework. Section 6 also discusses how the DDTC model can address some of the key challenges identified beforehand and how it contributes to narrowing the literature gaps. Furthermore, Section 6 presents the study's implications for the industry, its practitioners, and future research work. The originality of the paper provides an industry-specific analysis of the adoption of BCT coupled with a technological approach that leads to the development of a technological framework that provides key building blocks for industry practitioners to develop further and implement. 


\section{Methods}

At first, a traditional literature review method was used, and papers related to BCT, DT, and IoT were identified according to the research criteria presented in this section. BCT is a revolutionary technology allowing people to exchange value and data in a peer-to-peer way without trusting centralized institutions. Hence BCT will have a transformative effect on society as a whole and offer a paradigm shift on various dimensions of society, including key macro factors - such as the political, economic, social, technological, environmental, legal, and security (PESTELS) factors. Consequently, a PESTELS approach [2] was chosen to review the literature and extract key information from the selected papers into each of the PESTELS categories.

The general method for this paper is qualitative and pursued the following steps: database selection, search criteria, selection of relevant papers, the definition of the research directions, identification of key challenges, the identification of technological factors, and the extraction of technical gaps. Furthermore, additional relevant technical papers were selected in order to perform content analysis and extract state-of-the-art techniques to narrow the gaps identified.

The main databases were selected for their relevance to the engineering field: Compendex, Scopus, and Engineering Village.

The search criteria used were:

- Blockchain OR “distributed ledger" AND "construction" OR "construction industry" OR "built environment" OR "building industry" OR building OR "mine*" OR "mining";

- Blockchain OR "distributed ledger" AND "BIM";

- Blockchain OR "distributed ledger" AND "digital twin*" OR "cyber physical system*"; - Blockchain OR "distributed ledger" AND "smart building*" OR "intelligent building*" OR "smart home*" OR "Internet of Things" OR "IoT".

Papers were selected according to the quality of the journal (Q1 and Q2 journals prevailed over lower-level journals), the relevance to the topic, and the filtering by date of publication. BCT is in its infancy and evolves at a very fast pace. Thus, papers prior to 2017 were not selected except if their relevance was essential for this review. References are mostly dated from 2017, 2018, 2019, and a few recent papers from 2020 are also cited. Papers were selected based on their keywords, abstracts, and conclusions in terms of relevance to the topic. The main relevant keywords identified were: blockchain, smart contracts, distributed ledger, construction (industry), built environment, mining (industry), industry 4.0, smart building, intelligent building, smart home, BIM, digital twin, IoT, and cyber-physical systems. Most of the papers reviewed are key journal articles, with four conference papers also included. The papers were reviewed through a PESTELS approach, and the remaining references are related to specific technical papers selected for a content analysis of the blockchain technological state of the art for the purpose described in the preceding paragraph.

The PESTELS approach applied to review the literature permitted to extract key findings related to BCT adoption in the BECOM industry 4.0 for each PESTELS category. The information identified relates to industry-specific macro factors and to the key technologies: BIM, IoT, and DT. During the review of the papers, tables were produced to classify the extracted key information into every PESTELS category for each paper. It was then possible to identify and categorize the key challenges affecting BCT adoption in the BECOM industry 4.0 for each PESTELS category. From this categorization, the study then focused on the identified key technological (T) factors affecting the adoption of BCT and its relation to the key technologies: BIM, IoT, and DT.

Additional papers were further selected specifically for their relevance to the technological (T) factors identified; Hence the content analysis of the technological state of the art literature about BCT (whitepapers of key blockchain-based protocols) was performed to identify technical solutions aiming to address the key technological $(\mathrm{T})$ challenges and factors identified in the literature. Subsequently, the paper proposes a technological frame- 
work based on a conceptual model (DDTC) for the integration of BCT with DT. Additionally, from the technological factors affecting BCT adoption in the industry, it was then possible to extract the technological gaps relevant to the adoption of BCT for DT in particular. The study then articulates how some of the gaps identified are narrowed by the proposed DDTC framework.

\section{Literature Review}

The adoption of BCT in the BECOM industry 4.0 is promising to enhance trust, collaboration, efficiency, data sharing, and information security [7]. The existing literature offers key models such as "the blockchain of circular BIM Things" [13] and "the cup of water theory" [9], which both leverage BCT as a trusted container for information security for BIM and IoT. Naturally, this concept can be extrapolated from BIM and IoT to CPS [22], such as DT for which BCT could act as a core security component and as the layer of trust. However, several challenges affecting the adoption of BCT in the BECOM industry 4.0 should be considered first.

A PESTELS approach is used to review the literature and categorize the key challenges affecting BCT adoption for the BECOM industry 4.0. These key challenges identified in the literature are presented in the below paragraph and are more detailed in Table 1.

The political factors relate to the regulations of the BECOM industry and its limiting effects on the adoption of BCT due to a lack of regulations and policies for this new technology. Currently, the regulations on BCT are uncertain [2], and policy makers should admit the need for a new contractual and enforcement model leveraging BCT [4]. Hence, the BECOM industry needs regulatory changes to embrace digitization further [7]. The current regulatory framework originates from a centralized paper-based model that is disjointed from the blockchain paradigm shift that embraces the decentralization of services, and the automation of processes with smart contracts. A technological shift of the regulatory processes themselves would facilitate the adoption of BCT in the BECOM industry 4.0.

The economic factors refer to economic performances, efficiency, productivity, and costs in relation to the adoption of BCT. The productivity in the industry is stagnating [9], and efficiencies are generally low [22]. The financial ecosystem of the industry is generally fragile, and the pricing model is adversarial [7]. Moreover, financial transparency is lacking in the BECOM industry [2]. However, the high implementation cost of BCT is a key challenge for adoption as margins are low in the BECOM industry [7]. BCT may be costly to implement, however once adoption is generalized, the longer-term financial benefits for the BECOM industry 4.0 are likely to be significant and make the former centralized model obsolete due to overhead costs and inefficiencies.

The social factors refer to cultural aspects, workforce demographics, lack of skills, trust, industry habits, and attitudes that affect BCT adoption. The key social challenges for the BECOM industry are a poor industry image, its structural fragmentation, the lack of collaboration, lack of trust [23], and lack of transparency [22]. Indeed, the BECOM industry is fragmented [15] in data silos that restrict collaboration, limit trust in data governance [24], and create inefficiencies in information sharing and information management. The lack of trust in the industry leads to adversarial behaviors incentivizing stakeholders to hold information instead of sharing data for the benefit of the projects. BCT is decentralized, open, and secure by nature; moreover, it leverages cryptocurrencies as an incentivization mechanism to ensure that participants 'play by the rules' of the blockchain protocols consensus. Consequently, blockchain based systems could incentivize stakeholders of BECOM projects to share data in order to enhance collaboration in a competitive environment. Therefore, BCT is likely to optimize coopetition in the BECOM industry 4.0.

The technological factors refer to technological aspects, limitations, and requirements affecting the adoption of BCT for the BECOM industry 4.0. Some fundamental technological challenges for BCT adoption are the requirements for specific advanced IT infrastructures [22] and the need for efficient blockchain consensus mechanisms ensuring energy efficiency [23]. Moreover, the blockchain trilemma limits the capacity of a blockchain 
network to achieve at the same time decentralization, scalability, and security [25]. Other key challenges limiting BCT adoption relate to technical requirements of the BECOM industry 4.0, such as the interoperability between different blockchains [7], transaction privacy, network governance, and the integration of BCT with other key technologies of the industry such as IoT, BIM, and DT. Currently, DT solutions are too centralized, which induces single point of failure vulnerabilities. Additionally, data sharing for DT needs to be secured throughout the lifecycles of the physical asset that is represented by the DT [26]. There are key technological factors affecting BCT adoption for DT in the BECOM industry 4.0. Indeed, DT applications are typically centralized, leverage Big Data analysis and storage, and enable privacy as required with siloed databases and strict access control mechanisms. Whereas BCT is decentralized, has very limited storage capacity, and its openness by nature makes confidentiality a major challenge. Hence, critical trade-offs and state-of the-art techniques need to be explored and discussed to integrate BCT with DT in order to improve DTs security with blockchain.

The environmental factors refer to environmental sustainability, energy consumption, and recycling and reuse of materials. Materials wastes are a challenge for the BECOM industry [27]. The main environmental challenge for blockchain adoption is the energy consumption [7] of the consensus mechanism of certain public blockchain networks using proof-of-work (PoW) 'mining' which consumes large amounts of electricity. BCT can enhance waste management through traceability of materials along the lifecycle of projects. However, the carbon savings accomplished with materials recycling and reuse, should not be counterbalanced by the carbon emissions due to the energy consumption of blockchain based protocols. Hence, the energy efficiency of blockchain protocols is a critical factor to achieve substantial benefits for the environment in the BECOM industry 4.0.

The legal factors refer to contractual and legal aspects affecting the adoption of BCT in the BECOM industry. The key legal challenges in the BECOM industry relate to legal disputes, breaches of contracts, poor payment practices, and compliance issues [6]. The standards and regulations are stringent in the BECOM industry, but the certifications practices are substandard [21]. There is also a lack of standardization for BCT [26], and there are compliance challenges between BCT and the General Data Protection Regulation (GDPR) developed in Europe [28]. Moreover, proving ownership and liabilities is challenging [15], notably for the ownership of BIM models [4]. BCT and legal smart contracts can enforce accountability and non-repudiation through traceability of legal information, digital identities, and data ownership.

The security factors refer to the cyber security aspects that are affecting the adoption of $\mathrm{BCT}$ in the BECOM industry. With the digitization of the industry, cyber threats represent a primary risk for organizations [2]. Data privacy [22], data security, and data protection are major challenges of the industrial revolution 4.0 [1]. BCT is decentralized and secure by nature, hence BCT offers a paradigm shift from the existing security model of centralized infrastructure that are currently isolated in data silos secured by firewalls. Whereas BCT can guarantee security with trusted open networks that are secured by cryptography and public key infrastructure. This cyber security paradigm shift offered by BCT has considerable potential to decentralize and secure IoT networks [23] and DT data sharing [26] against malicious attacks.

Table 1 summarizes the PESTELS challenges affecting BCT adoption in the BECOM industry, that were identified through the literature review. 
Table 1. Summary of the challenges related to BCT adoption in the BECOM industry 4.0.

\begin{tabular}{|c|c|c|}
\hline PESTELS Challenges & & References \\
\hline Political challenges & & 5 references \\
\hline Uncertain regulations & P1 & [2] \\
\hline Inadequate regulatory oversight and enforcement tools & P2 & [7] \\
\hline Poor regulation and compliance & P3 & {$[1,7]$} \\
\hline Need for regulatory changes to adopt digitization & $\mathrm{P} 4$ & [7] \\
\hline Regulatory difficulties between BCT and GDPR & P5 & [23] \\
\hline $\begin{array}{l}\text { Policymakers and lawyers need to recognize the demand for a new } \\
\text { contractual model and to deliver it efficiently }\end{array}$ & P6 & [4] \\
\hline Economic challenges & & 7 references \\
\hline High implementation cost & E1 & {$[1,7,22]$} \\
\hline Lack of financial transparency & E2 & [2] \\
\hline Low efficiency & E3 & {$[6,9,22]$} \\
\hline Supply chain inefficiencies & $\mathrm{E} 4$ & [6] \\
\hline Stagnating/Low productivity of the construction industry & E5 & {$[4,7,9]$} \\
\hline Low predictability & E6 & [7] \\
\hline Low margins & E7 & [7] \\
\hline Increasing costs & $\mathrm{E} 8$ & [6] \\
\hline Adversarial pricing models and financial fragility & E9 & [7] \\
\hline Lack of R\&D and investments towards innovation & E10 & [7] \\
\hline Exchange rate volatility of cryptocurrencies & E11 & [7] \\
\hline Quality issues & E12 & [6] \\
\hline Delays & E13 & [6] \\
\hline Lose collaboration and inefficient management techniques & E14 & [9] \\
\hline Inefficient communication & E15 & [6] \\
\hline Limitations of the traditional funding and delivery model & E16 & [7] \\
\hline Poor supply chain management & E17 & [6] \\
\hline Social challenges & & 12 references \\
\hline Acceptance of new technologies & S1 & [1] \\
\hline Hesitation to adopt new technologies & $\mathrm{S} 2$ & {$[1,29]$} \\
\hline Lack of knowledge and skills regarding BCT and new technologies & S3 & {$[1,6,22]$} \\
\hline Cultural habits in the construction industry & S4 & [2] \\
\hline Lack of trust & S5 & {$[6,9,21,23]$} \\
\hline Lack of transparency & S6 & [22] \\
\hline Workforce size and demographics & S7 & [7] \\
\hline Lack of collaboration and lack of improvement culture & S8 & [7] \\
\hline Poor industry image & S9 & [7] \\
\hline Structural fragmentation of the industry & S10 & {$[6,7,15]$} \\
\hline Resistance to organizational and processes changes & S11 & {$[1,2,7]$} \\
\hline Lack of collaboration and trust in data governance & S12 & [24] \\
\hline Contractors acting as a central third parties & $\mathrm{S} 13$ & [4] \\
\hline Adversarial industry & S14 & [6] \\
\hline Dysfunctional training & S15 & [7] \\
\hline Slow adoption of new technologies by the construction industry & S16 & [7] \\
\hline Readiness for adoption of new technologies & S17 & [7] \\
\hline Technological challenges & & 14 references \\
\hline IT infrastructure requirements & & 5 references \\
\hline Higher requirements of $\mathrm{BCT}$ for computing equipment & $\mathrm{T} 1$ & [1] \\
\hline Need to enhance existing communication networks & $\mathrm{T} 2$ & [1] \\
\hline Blockchain requires specific IT infrastructure & $\mathrm{T} 3$ & {$[22,23]$} \\
\hline Storage capacity requirements for BCT & $\mathrm{T} 4$ & {$[22]$} \\
\hline Bandwidth and connectivity requirements for BCT & T5 & [7] \\
\hline Lack of standards and reference architectures & T6 & [1] \\
\hline Governance of networks & $\mathrm{T} 7$ & [2] \\
\hline Digital twin (DT) challenges & & 4 references \\
\hline Complex data specifications for DT (descriptive and sensor data) & $\mathrm{T} 8$ & [26] \\
\hline Need for DT throughout complex asset lifecycle & T9 & [21] \\
\hline Requirement to secure the data sharing of DT & $\mathrm{T} 10$ & [26] \\
\hline Current DT solutions are too centralized and rely on PLM system & $\mathrm{T} 11$ & [26] \\
\hline
\end{tabular}


Table 1. Cont.

\begin{tabular}{|c|c|c|}
\hline PESTELS Challenges & & References \\
\hline Need for a DT federated model aggregating all the models & $\mathrm{T} 12$ & [24] \\
\hline $\begin{array}{l}\text { Data storage requirements for the Big Data collected during DT } \\
\text { lifecycles }\end{array}$ & $\mathrm{T} 13$ & [24] \\
\hline $\begin{array}{l}\text { DT for multi-party sharing need to support data variety, data } \\
\text { velocity, data integrity, confidentiality, read and write operations }\end{array}$ & $\mathrm{T} 14$ & [26] \\
\hline $\begin{array}{l}\text { DT and IoT need high throughput and tamper resistant systems } \\
\text { (authenticity, provenance, proof of ownership, lifecycle traceability, } \\
\text { data input from IoT) }\end{array}$ & $\mathrm{T} 15$ & [24] \\
\hline Need for IoT enabled devices for each building system & $\mathrm{T} 16$ & [7] \\
\hline Data requirements for $\mathrm{DT}$ & & 9 references \\
\hline Data security & $\mathrm{T} 17$ & [1] \\
\hline Data format variety & $\mathrm{T} 18$ & [26] \\
\hline Data velocity & $\mathrm{T} 19$ & [26] \\
\hline Data volume & $\mathrm{T} 20$ & {$[15,24]$} \\
\hline Data ownership & $\mathrm{T} 21$ & {$[15,24,26]$} \\
\hline Data integrity & $\mathrm{T} 22$ & {$[6,22,23]$} \\
\hline Authentication and trustworthiness of the data & $\mathrm{T} 23$ & {$[7,22]$} \\
\hline Data availability & $\mathrm{T} 24$ & [23] \\
\hline Data privacy & $\mathrm{T} 25$ & [23] \\
\hline Data interoperability for information sharing & $\mathrm{T} 26$ & [6] \\
\hline Data management as per The Gemini Principles & $\mathrm{T} 27$ & [19] \\
\hline Traceability and transparency (non-functional requirements) & & 6 references \\
\hline Need for traceability and transparency of information & $\mathrm{T} 27$ & [21] \\
\hline Necessity to track components & $\mathrm{T} 28$ & {$[6,21]$} \\
\hline Need for immutable audit trails to track and trace & $\mathrm{T} 29$ & [24] \\
\hline $\begin{array}{l}\text { Lack of transparency and traceability in the construction supply } \\
\text { chain because of high number of participants and short timeframes }\end{array}$ & T30 & [9] \\
\hline Need for traceability, transparency & T31 & {$[6,21,22]$} \\
\hline Lack of real time information & $\mathrm{T} 32$ & {$[6]$} \\
\hline Lack of data traceability in the industry & T33 & [15] \\
\hline Identity management & & 2 references \\
\hline Need for decentralized identity management for humans or IoT & $\mathrm{T} 34$ & [24] \\
\hline Technical challenges for IoT: access control and authentication & $\mathrm{T} 35$ & [23] \\
\hline Decentralization (non-functional requirement) & & 3 references \\
\hline Industrial networks are too centralized & T36 & {$[22,23]$} \\
\hline Unnecessary trusted third parties in the industry & $\mathrm{T} 37$ & [22] \\
\hline $\begin{array}{c}\text { IoT management is limited because of centralized databases } \\
\text { systems vulnerable against attacks }\end{array}$ & $\mathrm{T} 38$ & [9] \\
\hline Blockchain performances challenges & & 6 references \\
\hline $\mathrm{BCT}$ has a low throughput compared to centralized databases & T39 & {$[6,7,23]$} \\
\hline Lack of scalability of BCT & $\mathrm{T} 40$ & {$[15,23]$} \\
\hline High latency of BCT & $\mathrm{T} 41$ & {$[6,7]$} \\
\hline Blockchain interoperability & $\mathrm{T} 42$ & {$[7,22]$} \\
\hline Proof-of-work (PoW) i.e., mining is not energy efficient & $\mathrm{T} 43$ & [23] \\
\hline Difficulty to develop new blockchain consensus mechanisms & $\mathrm{T} 44$ & [22] \\
\hline $\begin{array}{l}\text { Blockchain network access challenges (permissioned or } \\
\text { permission-less) }\end{array}$ & $\mathrm{T} 45$ & [22] \\
\hline Choice between public or private blockchain & $\mathrm{T} 46$ & {$[22]$} \\
\hline Security and tamper proof requirements for the industry & $\mathrm{T} 47$ & {$[22,23]$} \\
\hline $\begin{array}{l}\text { Blockchain scalability trilemma: trade-off between scalability, } \\
\text { decentralization, and security }\end{array}$ & $\mathrm{T} 48$ & [25] \\
\hline Privacy requirements for the industry & $\mathrm{T} 49$ & {$[22,23]$} \\
\hline Resilience against cyber-attacks is required in the industry & $\mathrm{T} 50$ & [22] \\
\hline Data storage limitations on the blockchain & $\mathrm{T} 51$ & {$[15,23]$} \\
\hline Asset's lifecycle complexity & & 4 references \\
\hline $\begin{array}{c}\text { Complexity of assets life cycles (design, build, inspect, monitor, } \\
\text { certify and manage) for DT }\end{array}$ & $\mathrm{T} 52$ & [21] \\
\hline $\begin{array}{l}\text { Broken information flow across lifecycle phases is a challenge for } \\
\text { DT that can be addressed by BCT }\end{array}$ & $\mathrm{T} 53$ & [26] \\
\hline
\end{tabular}


Table 1. Cont.

\begin{tabular}{|c|c|c|}
\hline PESTELS Challenges & & References \\
\hline $\begin{array}{l}\text { Traditional life cycle management (LCM) issues such as data } \\
\text { sharing and efficiency apply to building LCM through BIM }\end{array}$ & $\mathrm{T} 54$ & [9] \\
\hline $\begin{array}{l}\text { Difficulty to reuse existing blockchain network due to the one-off } \\
\text { nature of construction project }\end{array}$ & $\mathrm{T} 55$ & {$[6]$} \\
\hline BIM challenges & & 4 references \\
\hline Difficulty to track changes in BIM models & $\mathrm{T} 56$ & {$[15,30]$} \\
\hline $\begin{array}{l}\text { Attacks from the BIM central operator cannot be recorded and } \\
\text { tracked }\end{array}$ & $\mathrm{T} 57$ & [30] \\
\hline $\begin{array}{l}\text { Blockchain cannot scale in terms of storage for BIM data, it can only } \\
\text { record the digital proof }\end{array}$ & $\mathrm{T} 58$ & {$[15,30]$} \\
\hline $\begin{array}{l}\text { BIM model information is currently stored in data silos containing } \\
\text { different models' versions }\end{array}$ & T59 & {$[9,15]$} \\
\hline Difficulties to guarantee the integrity of historical BIM data & $\mathrm{T} 60$ & {$[30]$} \\
\hline $\begin{array}{l}\text { Lack of storage and scalability of BCT to run BIM model entirely on } \\
\text { BCT as a decentralized application }\end{array}$ & $\mathrm{T} 61$ & [7] \\
\hline Difficulty to achieve confidentiality with BCT & $\mathrm{T} 62$ & [15] \\
\hline Need for disintermediation in the industry & T63 & [15] \\
\hline Need for inter-organizational recordkeeping & $\mathrm{T} 64$ & [30] \\
\hline Smart contracts challenges & & 1 reference \\
\hline Difficulty to code smart contracts & $\mathrm{T} 65$ & [7] \\
\hline $\begin{array}{l}\text { Challenge to program bug free smart contracts which are } \\
\text { permanently stored on the blockchain }\end{array}$ & $\mathrm{T} 66$ & [7] \\
\hline Digitization challenges & & 3 references \\
\hline $\begin{array}{l}\text { Slow development of the AEC/FM (facility management) industry } \\
\text { due to slow/poor digitization }\end{array}$ & T67 & {$[7,9]$} \\
\hline Usage of different digital tools: interoperability issues & $\mathrm{T} 68$ & [26] \\
\hline Lack of adequate collaboration and information sharing & $\mathrm{T} 69$ & [7] \\
\hline Lack of a single source of truth in the AEC/FM industry & $\mathrm{T} 70$ & [9] \\
\hline Other challenges & & 4 references \\
\hline Technical challenges & $\mathrm{T} 71$ & [2] \\
\hline $\begin{array}{c}\text { Increased structural demand on buildings (similar to airplanes in } \\
\text { the aircraft industry) }\end{array}$ & $\mathrm{T} 72$ & [21] \\
\hline Need for systems flexibility & $\mathrm{T} 73$ & [22] \\
\hline $\begin{array}{l}\text { Off chain storage paired with distributed hash tables (DHT) for } \\
\text { each blockchain node }\end{array}$ & $\mathrm{T} 74$ & [26] \\
\hline Environmental challenges & & 3 references \\
\hline Energy consumption of BCT & EN1 & {$[7,22,23]$} \\
\hline Materials waste in the industry & EN2 & [27] \\
\hline Legal challenges & & 11 references \\
\hline Standards and Compliance & & 4 references \\
\hline Lack of standards for blockchain & L1 & [26] \\
\hline Stringent standards, regulations, and certifications in the industry & $\mathrm{L} 2$ & [21] \\
\hline Legal disputes and compliance issues & L3 & {$[6,22]$} \\
\hline Contractual & & 6 references \\
\hline Legal and contractual uncertainty & L4 & [1] \\
\hline $\begin{array}{l}\text { Traditional contracts are time consuming and involve several } \\
\text { parties }\end{array}$ & L5 & [9] \\
\hline Contracts breaches are frequent in the industry & L6 & [6] \\
\hline $\begin{array}{l}\text { Poor payment practices (late payments, not paid, held up } \\
\text { payments) and associated contractual issues }\end{array}$ & L7 & [7] \\
\hline Non-repudiation & L8 & {$[15,23]$} \\
\hline Ownership and liabilities & & 4 references \\
\hline $\begin{array}{c}\text { Difficulty to prove intellectual property rights (ownership, } \\
\text { immutability) }\end{array}$ & L9 & {$[15,24]$} \\
\hline BIM model ownership is an issue for legal disputes & L10 & {$[4,15]$} \\
\hline Lack of clarity on roles and responsibilities & L11 & {$[7,15]$} \\
\hline $\begin{array}{l}\text { Lack of enforceability is a key challenge as it is very difficult to } \\
\text { know who to hold accountable }\end{array}$ & L12 & {$[7]$} \\
\hline
\end{tabular}


Table 1. Cont

\begin{tabular}{|c|c|c|}
\hline PESTELS Challenges & & References \\
\hline Security challenges & & 8 references \\
\hline Data security and data protection & SE1 & [1] \\
\hline $\begin{array}{c}\text { Management and security of information shared within the supply } \\
\text { chain }\end{array}$ & SE2 & {$[24]$} \\
\hline $\begin{array}{l}\text { Current security methods need to build barriers / firewalls around } \\
\text { IT infrastructures }\end{array}$ & SE3 & {$[30]$} \\
\hline Need for a dynamic and adaptable security framework & SE4 & [23] \\
\hline Blockchain based IoT security and privacy & SE5 & [23] \\
\hline Need for data privacy & SE6 & {$[9,22]$} \\
\hline Centralized third parties are vulnerable as single points of failure & SE7 & [9] \\
\hline Need for privacy aware distributed hash table (DHT) & SE8 & [31] \\
\hline Resilience against combined attacks is a challenge for BCT and IoT & SE9 & [23] \\
\hline Cyber security risks & SE10 & [2] \\
\hline Malicious attacks/hacking of IoT & SE11 & {$[7,23]$} \\
\hline
\end{tabular}

The PESTELS categorization (summarized in Table 1) of the key challenges in the industry affecting BCT adoption satisfies the first objective of the paper. It should be noted that the technological $(\mathrm{T})$ challenges affecting BCT adoption are subdivided in Table 1. as follows: IT infrastructure, BIM, digital twin (DT), data requirements for DT, traceability and transparency, decentralization, identity management, blockchain performances, smart contracts, asset's lifecycle complexity, and digitization of the industry.

\section{Key Technological Factors Affecting Adoption}

As mentioned in the method section, a content analysis of the state-of-the-art literature on BCT was also performed in parallel. From this technical content analysis, the key technological (T) factors affecting BCT adoption in the BECOM industry 4.0 (shown in Table 1) were reorganized logically in key categories consistent with the key technical challenges and taxonomy around BCT. The categories of key technological (T) factors affecting the adoption of BCT in the BECOM industry 4.0 are presented in Table 2, which frame the validation of the second objective of this paper. The key technological factors shown in Table 2 are the IT requirements for BCT, the technical requirements for BCT applications, and the integration of BCT with other technologies (BIM, IoT, and DT) of the BECOM industry 4.0. In order to fully satisfy the second objective of this paper, the technological factors are explained further in Section 4. The third column of Table 2 compares the technological factors with key related use cases and technological components obtained from both the literature review and the content analysis of the state-of-the-art literature on BCT. This identification of key technological factors sets the foundations and provides the building blocks for the technological framework proposed in Section 5. 
Table 2. Key technological factors affecting the adoption of blockchain technology in the BECOM industry.

\begin{tabular}{|c|c|c|c|}
\hline Group & & $\begin{array}{l}\text { Key Technological } \\
\text { Factors }\end{array}$ & Uses Cases and Technological Components References \\
\hline \multirow[t]{3}{*}{$\begin{array}{l}\text { A. IT } \\
\text { requirements }\end{array}$} & 1 & Decentralization & $\begin{array}{c}\text { Open-source code for BCT protocols [11,32]. } \\
\text { Decentralized payments and smart contracts to automate processes [4]. } \\
\text { Decentralized applications (DApp) and decentralized autonomous } \\
\text { organizations (DAO) which could be integrated with BIM [9]. } \\
\text { BCT removes intermediaries [7]. } \\
\text { BCT eliminates the need for security barriers which generate data silos } \\
\text { [30]. }\end{array}$ \\
\hline & 2 & $\begin{array}{l}\text { IT infrastructure and } \\
\text { computational } \\
\text { requirements }\end{array}$ & $\begin{array}{l}\text { Distributed computing solutions such as iExec [33] or Dfinity [34] } \\
\text { could compute decentralized applications of the BECOM industry. } \\
\text { Off chain decentralized data storage could be mapped to blockchain } \\
\text { through distributed hash tables (DHT) [26]. } \\
\text { Decentralized storage [23] such as Storj [35] and distributed storage, } \\
\text { such as Swarm [36] or IPFS [37], could be suitable when they become } \\
\text { adequately scalable for applications of the BECOM industry like BIM. }\end{array}$ \\
\hline & 3 & Big Data requirements & $\begin{array}{l}\text { BCT would need to be adequate for data variety, data velocity [26], and } \\
\text { data volume [15]. } \\
\text { To guarantee data integrity from IoT devices, data should be } \\
\text { authenticated using crypto hardware secure element [38] before } \\
\text { entering the blockchain to avoid "garbage in, garbage out" (GIGO) } \\
\text { effects. } \\
\text { Data interoperability is required for the information sharing industry } \\
\text { [6]. } \\
\text { Data security and privacy are key requirements for the BECOM } \\
\text { industry } 4.0[9,22,23] . \\
\text { Real-world project data could be input to the blockchain through } \\
\text { decentralized oracles like Chainlink [39]. }\end{array}$ \\
\hline \multirow[t]{2}{*}{$\begin{array}{l}\text { B. Blockchain } \\
\text { applications } \\
\text { requirements }\end{array}$} & 4 & $\begin{array}{l}\text { Smart contracts } \\
\text { integration }\end{array}$ & $\begin{array}{c}\text { Disintermediate and automate processes [22]. } \\
\text { Release payments automatically when tasks are completed [4]. } \\
\text { Manage project bank accounts [7]. } \\
\text { Save time, increase efficiency, reduce manual errors, record BIM } \\
\text { operations and allocate ownership and responsibilities, secure } \\
\text { transactions of properties, land ownership, equipment } \\
\text { leasing/purchase, and procurement, facilitate IoT management } \\
\text { challenges [9]. } \\
\text { Enable DApps [23] and DAOs [9]. } \\
\text { The Ethereum platform [32] integrates smart contracts, has a major } \\
\text { public blockchain, and its protocol can also be leveraged for } \\
\text { consortium and private blockchain networks. }\end{array}$ \\
\hline & 5 & $\begin{array}{c}\text { Identity } \\
\text { management/access } \\
\text { control }\end{array}$ & $\begin{array}{c}\text { The uPort project [40] offers a blockchain-based decentralized identity } \\
\text { service for the citizens of Zug in Switzerland. } \\
\text { The ERC-725 project is a blockchain-based self-sovereign identity } \\
\text { standard on Ethereum [41]. } \\
\text { To enhance privacy, zero-knowledge proof can be used for community } \\
\text { self-sovereign identity [42], for IoT devices identities [43], and to } \\
\text { manage access control for DHT [31]. } \\
\text { Identity authentication for IoT devices can be achieved with crypto } \\
\text { hardware such as the secure element by Riddle and Code [38]. }\end{array}$ \\
\hline
\end{tabular}


Table 2. Cont

\begin{tabular}{|c|c|c|c|}
\hline Group & & $\begin{array}{c}\text { Key Technological } \\
\text { Factors }\end{array}$ & Uses Cases and Technological Components References \\
\hline \multirow{3}{*}{$\begin{array}{l}\text { C. Blockchain } \\
\text { integration } \\
\text { with other } \\
\text { technologies }\end{array}$} & 6 & $\begin{array}{l}\text { Blockchain properties } \\
\text { and performances }\end{array}$ & $\begin{array}{c}\text { The scalability trilemma [25] claims that there is a trade-off between the } \\
\text { three key requirements of a blockchain network: scalability, } \\
\text { decentralization, and security. } \\
\text { Private, consortium, and public [30]. } \\
\text { Proof of Authority (PoA) consensus mechanism [44] for private and } \\
\text { consortium blockchains. } \\
\text { Governance of private nodes with Infrachain [40]. } \\
\text { Anchorage of dataset's hash proof to overcome scalability challenges [40]. } \\
\text { Proof of Stake (PoS) consensus mechanisms for public blockchains like } \\
\text { Ethereum 2.0 [45] with Casper [46] or Cardano [47] with Ouroboros } \\
\text { [48] which are more energy efficient than proof-of-work (PoW). } \\
\text { Blockchain privacy projects: Aztec [49], Enigma [50], and the Baseline } \\
\text { protocol [51]. } \\
\text { Blockchain interoperability projects: Cosmos [52], Interledger [53], } \\
\text { Polkadot [54], Quant [55], or APIs and Oracles [56]. }\end{array}$ \\
\hline & 7 & $\begin{array}{c}\text { Blockchain integration } \\
\text { with BIM }\end{array}$ & $\begin{array}{l}\text { Blockchain can provide immutable records for the sharing of historical } \\
\text { BIM data [30] in a trusted collaborative environment [9]. } \\
\text { Real-time model updates from network participants based on their } \\
\text { access rights [7]. } \\
\text { Signing of BIM models with the BIMCHAIN software [57]. } \\
\text { BIM Level } 3 \text { and BCT can bring a single shared source of truth of the } \\
\text { BIM model in a distributed, trusted collaborative environment [9]. } \\
\text { BIM Level } 3 \text { complies with Industry Foundation Classes (IFC) and } \\
\text { BuildingSMART Data Dictionary (bsDD), which could be integrated to } \\
\text { BCT [7] through a standardized data structure, with hash proof of BIM } \\
\text { data, recorded into the blockchain blocks [30]. } \\
\text { New BIM dimensions could be considered [58] in relation to BCT such } \\
\text { as a financial dimension enabled by decentralized blockchain } \\
\text { payments or a contractual dimension through smart contracts. } \\
\text { To overcome data storage limitations on the blockchain, distributed } \\
\text { hash tables (DHT) could be used [23] to map the blockchain hash } \\
\text { proofs to cloud storage. } \\
\text { Decentralized [35] and distributed storage [36] will likely become } \\
\text { adequately scalable for a large volume of BIM data. }\end{array}$ \\
\hline & 8 & $\begin{array}{c}\text { Blockchain integration } \\
\text { with IoT }\end{array}$ & $\begin{array}{l}\text { BCT can defragment IoT ecosystems through decentralization of the } \\
\text { IoT networks' management [9]. } \\
\text { Network of validators nodes for IoT devices should be sufficiently } \\
\text { distributed as per the INCUBED protocol [59]. } \\
\text { IoT data collected through the value chain can update the ledger, } \\
\text { providing a single source of truth to track components, avoid } \\
\text { duplication of work and improve facility management [7]. } \\
\text { Blockchain can act as a secure and transparent container for historical } \\
\text { IoT data [9]. } \\
\text { Ethereum, Hyperledger Fabric, and IOTA are suitable DLT for IoT [60]. } \\
\text { Ethereum blockchain is public and has a native cryptocurrency Ether } \\
\text { (ETH), whereas Hyperledger is not public and does not have a native } \\
\text { currency. Ethereum enables smart contracts while IOTA do not. } \\
\text { However, Ethereum requires a transaction fee on its public network, } \\
\text { whereas IOTA does not. } \\
\text { Crypto hardware secure elements [38] embedded into IoT devices can } \\
\text { authenticate IoT devices and IoT data to enhance data integrity. } \\
\text { Resource-constrained IoT devices can offload computation to master } \\
\text { validating nodes [60] using fog computing at the edge of the network [61]. } \\
\text { Energy harvesting for IoT devices is integrated to mining equipment so } \\
\text { that IoT devices batteries can be recharged using the vehicle's } \\
\text { vibrations [62]. }\end{array}$ \\
\hline
\end{tabular}


Table 2. Cont.

\begin{tabular}{|c|c|c|c|}
\hline Group & & $\begin{array}{l}\text { Key Technological } \\
\text { Factors }\end{array}$ & Uses Cases and Technological Components References \\
\hline & 9 & $\begin{array}{l}\text { Blockchain integration } \\
\text { with digital twin }\end{array}$ & $\begin{array}{l}\text { DLT is suitable to secure DT data [26]. } \\
\text { Decentralized common data environment (DCDE) [9]. } \\
\text { Distributed collaborative environment can benefit the project } \\
\text { information model (PIM) and the asset information model (AIM) } \\
\text { [63] throughout the lifecycle of the asset and its DT. } \\
\text { Big Data from the project information value chain should be } \\
\text { managed in accordance with The Gemini Principles in order to } \\
\text { reduce data volume and increase data value [19] } \\
\text { IoT, BIM, and blockchain can also enhance real-time monitoring for } \\
\text { construction processes and management of facilities [7]. } \\
\text { A blockchain-enabled CPS architecture-i.e., BCPS-could use } \\
\text { three layers [22]: the cyber net in the back end, the connection net } \\
\text { for the sensing network of IoT, and the data monitoring } \\
\text { management net presenting data insights to end-users through } \\
\text { front end applications. } \\
\text { Analogy with the "cup of water" theory [9], DTs represent the } \\
\text { foundation ("cup base") of digitization for the BECOM industry 4.0, } \\
\text { IoT collect authenticated real-world data flow ("water") throughout } \\
\text { the projects value chain, and blockchain acts as the "cup wall" } \\
\text { containing the data securely and efficiently in a transparent way. }\end{array}$ \\
\hline & 10 & $\begin{array}{l}\text { Asset lifecycle } \\
\text { complexity }\end{array}$ & $\begin{array}{c}\text { DLT can unify the fragmented information flow across phases of } \\
\text { the projects' lifecycle [26]. } \\
\text { BECOM industry projects data can be recorded on the blockchain } \\
\text { throughout the various phases of their lifecycle: design, } \\
\text { manufacturing, materials delivery/procurement, construction } \\
\text { processes, inspection certification, QA, asset management, } \\
\text { decommissioning, and demolition [21]. } \\
\text { Blockchain-based information management throughout the } \\
\text { lifecycle of the asset's digital twin can benefit the CE [26] and } \\
\text { improve sustainability [64] as it facilitates traceability of materials } \\
\text { for reuse and recycling. }\end{array}$ \\
\hline
\end{tabular}

The key technological factors affecting BCT adoption shown in Table 2 are grouped into the three key categories A, B, and C that is discussed as follows.

Table 2 refers to the three main factors related to information technology (IT) requirements (Group A). The first IT requirement is to achieve adequate decentralization of networks in the industry since decentralization is at the essence of BCT that provides an immutable trusted distributed ledger (through peer-to-peer networks) without relying on centralized trusted third parties [9]. The second IT requirement relates to the decentralization of IT infrastructures and computational capacities using, for example, decentralized trusted off-chain computing [33] and decentralized storage systems [35]. Thus, decentralized applications (DApps) and data storage requirements for BECOM industry applications would not rely on centralized IT systems that are vulnerable to cyber threats. In terms of data storage, off-chain decentralized storage could be mapped to the blockchain using systems like distributed hash tables (DHT) [26]. The third IT requirement is the integration of $\mathrm{BCT}$ with the data value chain of the BECOM industry. Big Data transacting throughout $\mathrm{BECOM}$ projects' lifecycles follows the $3 \mathrm{Vs}$ characteristics comprising the variety of data formats (CAD, BIM, GIS, IFC, XML, COBie, planning, design cost, IoT, RFID, survey, asset information), data velocity, and data volume. Moreover, data authentication, integrity, interoperability, security, and privacy are key non-functional requirements to address for the data value chain in the industry. Adequate data structures, schemas, and standardizations are required to integrate an authenticated, structured, and trusted data layer into the blockchain from the project value chain. 
Table 2 refers to three key requirements for blockchain (Group B) applications in the BECOM industry 4.0. Blockchain smart contracts are programmable transactions that can automate key processes such as business logic, and are executed in a decentralized way. Hence, smart contracts have great potential to automate processes and remove middlemen [22] in the BECOM industry 4.0. The integration of smart contracts is a key requirement for blockchain applications BECOM industry 4.0 as presented in Table 2. Smart contracts can also contain hash values guaranteeing the integrity of sensors data stored off the chain [26]. The second requirement is a digital identity and access control framework that is required to authenticate projects' stakeholders and IoT devices transacting through blockchain applications. IoT devices identities can be protected with crypto hardware secure elements [38] to guarantee data authentication at the source. The third requirement for blockchain applications in the BECOM industry 4.0 relates to key non-functional requirements for blockchain networks which are: scalability, security, decentralization, interoperability, privacy, governance, and energy efficiency. As mentioned previously, the blockchain trilemma [25] means that the scalability, security, and decentralization of the network cannot be satisfied altogether without one being compromised. Hence trade-offs are required between these three fundamental properties when designing blockchain applications. Finally, in terms of blockchain governance, there are three types of blockchain networks to consider: private, consortium, and public [30]. Privacy is also a key technological (T) factor affecting the adoption of BCT applications in the BECOM industry. It is naturally easier to achieve privacy with private blockchains, whereas it is a key challenge for public blockchains to enable private transactions. Achieving complete privacy on public blockchain networks appears counter-intuitive; however, privacy protocols like Aztec [49] using zero-knowledge proofs, Enigma [50], the Baseline protocol [51], or encryption mechanisms could be leveraged. Finally, interoperability between blockchains is a key technological (T) factor affecting BCT adoption in the BECOM industry due to the possibility of many stakeholders using blockchain applications built on different blockchain networks but that still require transacting together. Figure 1 considers the interoperability requirement between the stakeholders' blockchain ledgers within the BECOM industry.

Table 2 shows that there are concerns in the literature about the integration of BCT with the other key technologies of the BECOM industry 4.0 like BIM, IoT, and DT (Group C). BIM historical data and model changes can be recorded immutably on blockchain distributed ledgers [30]. Hence, BCT can enhance trust and transparency, prove ownership, responsibilities, liabilities, and intellectual property rights for BIM [7]. In addition, smart buildings' IoT sensors should inform the model about maintenance requirements in realtime [4]. The historical data of smart building assets should be recorded on the blockchain. However, blockchain has very limited storage capacity, making it practically impossible to store BIM and IoT data. Anchoring hash proofs of BIM data into the blockchain blocks [30] is an alternative method to overcome blockchain storage limitations. BIM Level 3 complies with Industry Foundation Classes (IFC) and BuildingSMART Data Dictionary (bsDD), which needs to be integrated into BCT [7] through a standardized data structure and data schemas. Other key challenges for the integration of BCT and IoT are the authentication of IoT devices and IoT data [22], the need to decentralize IoT networks' management [9], and resource-constrained IoT devices which have the limited computational capacity to operate full blockchain nodes [60]. Regarding DT, blockchain needs to provide a verified data audit trail and bring trust and transparency to DT ecosystems [20] throughout the project's complex lifecycle over a decentralized common data environment (DCDE) [9]. 


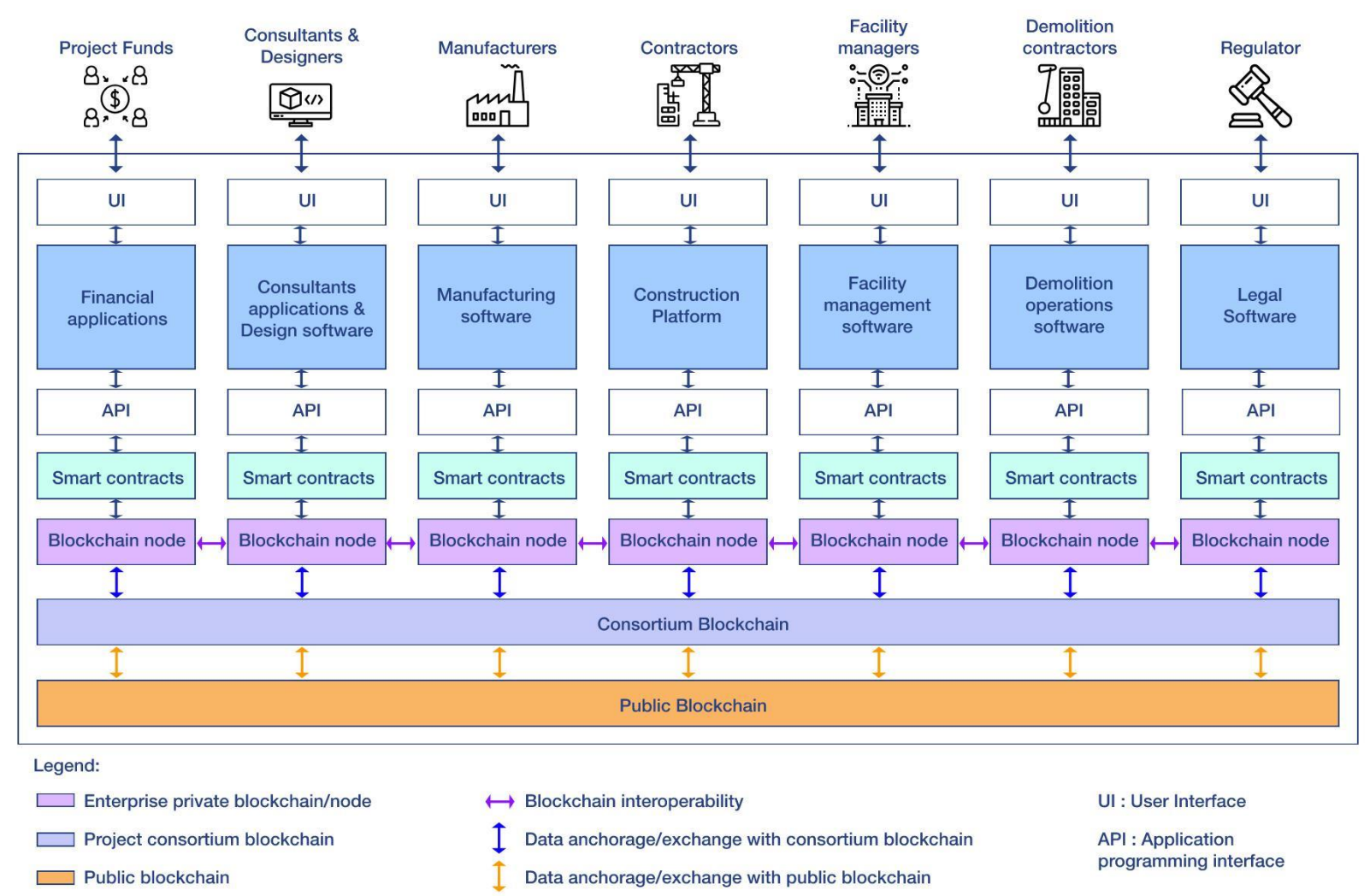

Figure 1. Blockchain ecosystem diagram between industry stakeholders at different levels: enterprise, consortium, and public. Adapted from Figure 10 of the World Economic Forum. Inclusive Deployment of Blockchain for Supply Chains: Part 6-A framework for Blockchain Interoperability; 2020 [56].

The technological (T) factors described above affect the integration of BCT with DT, which is a key technology of the BECOM industry 4.0 composed of IoT, BIM, data storage systems, computing layers, and Big Data flowing from the project information value chain. Since this study focus particularly on the combination of BCT and DT in the BECOM industry 4.0, the following section proposes a technological framework based on a novel conceptual model combining BCT and DT. The key technological factors presented in Table 2 and discussed in Section 4 provide the technological building blocks for the framework proposed in Section 5.

\section{Decentralized Digital Twin Cycle}

The paper offers the decentralized digital twin cycle (DDTC) conceptual model, comprising three core blockchain layers. BCT is at the core of the model, acting as the data integrity enabler that securely records key historical proofs for data transacting with DT throughout the lifecycle of projects in the BECOM industry 4.0. The DDTC has four key architectural layers: the connection layer, the data management layer, the computing layer, and the blockchain layer. Data from the BECOM project enters the DDTC through the external connection layer, which leverages software APIs (application programming interface), middleware, IoT devices, and RFID to capture "real world" project data across the various phases the project lifecycle. The information from the project data value chain enters the DDTC, and the data management layer comprises the information management framework in conformity with The Gemini Principles [19]. The data management layer also contains the DT applications user interfaces (front-end UI) for project participants to interact with the DT systems according to their access rights. The computation of the DDTC occurs within the computing layer. Ultimately, the blockchain core layer contains the project historical proofs of key data transacted with DT applications through immutable blockchain ledgers guaranteeing data integrity. 
The DDTC model was developed by considering the nine key technological factors (A.1, A.2, A.3, B.4, B.5, B.6, C.7, C.8, C.9) presented in Table 2 in Section 4. The following chapters explain how each of the technological factors is considered by the DDTC framework.

In terms of IT requirements, the computing layer of the DDTC framework embraces decentralization in order to remove intermediaries and reduce data silos (key factor A.1). For this purpose, it leverages distributed blockchain ledgers and decentralized applications (DApps, DAOs) programmed with an open source 'culture' for smart contracts code. The IT infrastructure of the DDTC also aims to be decentralized (key factor A.2) by leveraging distributed trusted computing protocols [33] and distributed storage systems [35], both running off the blockchain. Despite being 'off chain', these distributed computing and storage protocols still leverage BCT for security and access control to guarantee cyber resilience. The DDTC technological framework also needs to interoperate with Big Data from the information value chain of BECOM projects (key factor A.3). Middleware solutions contribute to standardizing the data structure before it can enter the back-end components. The framework also leverages decentralized oracles [39] protocols to authenticate external data (from IoT sensors, external APIs, or from various web sources) before inputting them into the blockchain layer. Once the correct data enters the blockchain, they become secure, and data integrity is maintained over time. Finally, the model considers the data privacy requirement through the use of private blockchains, privacy protocols [49,51], and encryption mechanisms.

In terms of BCT requirements, the integration of smart contracts (key factor B.4) is a key component of the DDTC computing layer to automate processes, increase efficiency, trace accountability, and enable DApps. Smart contracts would be programmed to compute key processes along the project lifecycle. The DDTC model also leverage decentralized digital identities (key factor B.5) such as self-sovereign identities [42] for project stakeholders and devices identities. Decentralized identities would improve access control mechanisms for DApps of the DDTC framework. In terms of blockchain properties and performances (key factor B.6), the DDTC suggests combining private blockchains, consortium blockchains, and public blockchains. Private blockchains would be suitable at the enterprise level, consortium blockchains would be adequate for an overall project ecosystem (e.g., for joint ventures), and public blockchains would be suitable for the data required to be openly available for the public auditability. At the enterprise level, employees could transact certain key information (e.g., BIM historical data, design parameters, IoT data, contracts, financial transactions, workflows, and approvals) through the enterprise private blockchain network. This enterprise-wide shared ledger would represent a single source and reduce data silos internally. The transaction throughput of a private blockchain is highly scalable, and there is no transaction fee. At the project level, cooperating organizations would transact data together via a consortium blockchain adequately interoperable with the enterprises' private networks, shown in Figure 1. The transaction throughput of consortium blockchains would be fast or moderate, depending on the type of data and the settlement finality requirements. When large volumes of data need to be transacted efficiently, settlements by packet could ensure that only key states hashes (digital footprints) are anchored in the shared ledger without transacting the actual datasets that can be stored off the blockchain. The transaction cost of such a consortium blockchain would be negligible and minimize the overhead costs of the project. The governance framework for private or consortium nodes could be achieved with systems like Infrachain [40]. At the public level, the projects stakeholders, and public external stakeholders (e.g., consumers and users) could transact and audit key data through a public blockchain that is open by nature. As such, public blockchains could serve as public registries that record transactions proofs [40] about key auditable data such as certificates, land registers, and environmental records. The transaction confirmation time and transaction cost for such a public blockchain would require relatively low for a viable user experience. Figure 1 illustrates this proposed mixed blockchain ecosystem combining private, consortium, and public blockchain for projects of the BECOM industry. 
The transactions that require high throughput and privacy at the enterprise or project level can be processed respectively on the private and consortium networks. Only data requiring public audibility can be anchored on the public blockchains. Hence, the DDTC aims to address the blockchain trilemma with the combination of interoperable private, consortium, and public blockchains. Consequently, the scalability requirements of the project can be addressed by the proposed model.

In terms of BCT integration with other key technologies of the BECOM industry 4.0, the DDTC model connects BCT to BIM, IoT, and DT. The DDTC model aims to record in the blockchain the key historical data proofs from these technologies at each phase of the project to provide a single source of truth. BCT can be integrated with BIM (key factor C.7) in order to anchor key BIM data on the blockchain (on chain). For example, at the design phase, 3D BIM spatial data, real-time models' updates, and design parameters could be anchored on the blockchain ('on-chain'). 4D BIM scheduling, procurement, and supply chain data could be anchored on the chain during construction. During operation and maintenance, 6D BIM data, smart building IoT sensors data, and maintenance data could be anchored on-chain. As shown in Figure 2, the data management layer of the DDTC comprises the key processes that could be automated with blockchain smart contracts as well as the key data that could be anchored on-chain. Future research work should identify what project data exactly should be stored on-chain or off-chain. As mentioned in the previous chapters, the DDTC model contains decentralized storage systems to also preserve decentralization for the data stored off-chain. To integrate BCT with IoT (key factor C.8), the DDTC model aims to decentralize the connection layer with blockchain-based protocols for IoT management. For this purpose, the DDTC model promotes the use of edge computing systems (on-premises computing servers), enabling computation power near the IoT devices (at the edge) [61] to optimize the analysis of the data captured from IoT sensors. Moreover, having edge computing resources can allow resource constraint IoT devices to indirectly run blockchain nodes by delegating the computational effort to master nodes [60]. The connection layer of the DDTC model includes secure elements [38] and microchips [43] to authenticate devices identities and enable data authentication at the source to limit the GIGO effects. Hence, the initial data authentication and curation occurring at the edge enables the filtering of the data entering the DDTC to reduce volume and increase value according to The Gemini Principles, as shown in Figure 2 at the junction between the connections and the computing layer. Furthermore, the inclusion of blockchain-based protocols in the connection layer facilitates the decentralization of IoT management and reduces single points of failure. Finally, the DDTC model proposes to combine BCT with DT (key factor C.9) to improve information management throughout the complex lifecycle of a project by unifying fragmented data silos towards a single source of truth with key data securely anchored in the blockchain.

The DDTC model addresses the key technological factors (A.1, A.2, A.3, B.4, B.5, B.6, C.7, C.8, C.9) identified in Section 4. Moreover, the DDTC model facilitates collaboration through peer-to-peer distributed networks and benefits the $\mathrm{CE}$ through materials traceability, reuse, and recycling.

The diagram in Figure 2 represents the proposed technological framework based on the decentralized digital twin cycle conceptual model. The core blockchain layer comprises a combination of the private, consortium, and public blockchains, as described in the previous chapter and illustrated in Figure 1.

The following Section 6 discusses the contributions and implications of the DDTC framework as well as the associated gaps identified in the literature in relation to combining these technologies. 


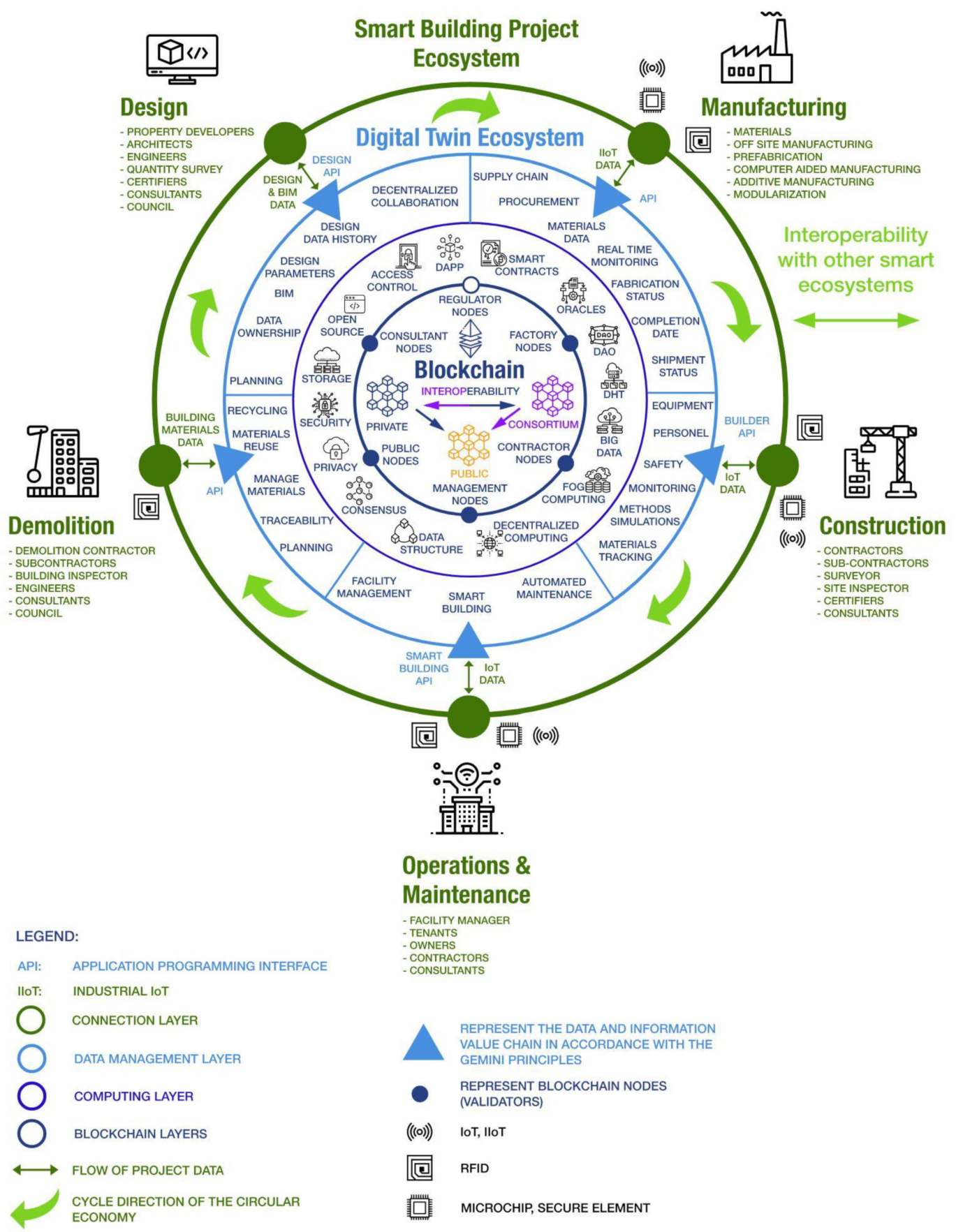

Figure 2. The decentralized digital twin cycle.

\section{Discussion}

The first contribution of the paper is a review of the literature following a PESTELS approach to identify the challenges affecting BCT adoption in the BECOM industry in all PESTELS categories. Hence, this approach is original since it identifies the key challenges related to the political ( 6 challenges), economical (17 challenges), social (17 challenges), technological (74 challenges), environmental ( 2 challenges), legal (12 challenges), and security (11 challenges) aspects as presented on Table 1 . The paper then focuses on the technological factors which are predominant and form the main objectives of the study. The industry-specific technological challenges identified in the literature were then compared with the key technical blockchain solutions and protocols identified through the content analysis of the state-of-the-art literature on BCT. This allowed us to isolate the key technological factors affecting adoption and compare them with key use cases and technological 
components in Table 2. This technological framework composed of key technological factors affecting adoption represents the second contribution of the paper. As presented in Table 2 of Section 4, nine key technological factors (A.1, A.2, A.3, B.4, B.5, B.6, C.7, C.8, C.9) were identified and categorized in three groups as follows: IT requirements (group A), blockchain applications requirements (group B), and the integration of blockchain with other technologies (group C).

Since BCT is not a mature technology yet, there are remaining technological limitations affecting its adoption for DT in the BECOM industry 4.0. Hence, from the key technological factors presented in Table 2, the study identified some key technology gaps areas in relation to BCT adoption for DT. These technological gaps should be addressed with future research work and the technological progress of BCT. Hence, the third contribution of the study is the identification of these technological gaps in relation to the adoption of BCT for DT in the BECOM industry 4.0. Indeed, the main focus of the paper was narrowed to five key technological gap areas contextualized to the adoption of BCT for DT in the BECOM industry 4.0. As shown on Table 3, the five gap areas identified in the literature are the technical and non-functional requirements of blockchain technology (gap area 1), technical limitations of IoT and blockchain technology association (gap area 2), BIM and blockchain challenges (gap area 3), digital twin data challenges with blockchain (gap area 4), and the complexity of projects lifecycles (gap area 4). These gaps areas cover a total of 14 themes for future research, as presented in Table 3. Finally, the main contribution of this paper is the development of a technological framework based on a conceptual model called the DDTC as presented in Section 5 and illustrated on Figure 2. Hence the theoretical contribution of the paper is linked to the proposed DDTC theoretical framework comprising the key technological components, and the sustainability and regulatory considerations that can be used by industry practitioners to develop the system and implement it. The DDTC model integrates technological components that contribute to narrowing some of the gaps identified. The content analysis of the technological state of the art on BCT permitted identifying potential solutions and protocols to address key technological factors and narrow the gaps as presented in the chapters below and summarized in the discussion column of Table 3. Finally, the contributions of the paper address the research questions raised in Section 1.

Table 3. Technical gaps analysis and themes for future investigations.

\begin{tabular}{|c|c|c|c|}
\hline Area of the Gap & Themes for Future Research & $\begin{array}{l}\text { PESTELS } \\
\text { Factors }\end{array}$ & Discussion on the Themes \\
\hline \multirow{3}{*}{$\begin{array}{l}\text { 1. Technical and } \\
\text { non-functional } \\
\text { requirements of } \\
\text { blockchain technology }\end{array}$} & $\begin{array}{l}\text { 1.1. Governance of the } \\
\text { blockchain network }[7,40]\end{array}$ & $\begin{array}{l}\mathrm{P} \\
\mathrm{T} \\
\mathrm{L}\end{array}$ & $\begin{array}{c}\text { Project stakeholders, including regulators, should run } \\
\text { independent blockchain nodes. } \\
\text { Validators' nodes can vote on key governance decisions. } \\
\text { Infrachain project [40] offers a governance solution for } \\
\text { private nodes. } \\
\text { Explore decentralized governance models for } \\
\text { blockchain networks. }\end{array}$ \\
\hline & $\begin{array}{l}\text { 1.2. Type of blockchain } \\
\text { network: private, consortium } \\
\text { and public }[7,22]\end{array}$ & $\begin{array}{l}\mathrm{P} \\
\text { Soc. } \\
\mathrm{T}\end{array}$ & $\begin{array}{c}\text { The decentralized digital twin cycle model proposes to } \\
\text { use private blockchains at the enterprises level, } \\
\text { consortium blockchain at the project level and public } \\
\text { blockchain to anchor key states for public auditability. } \\
\text { The integration of these three types of blockchain } \\
\text { networks for a project ecosystem is inexperienced, } \\
\text { complex, and needs to overcome interoperability } \\
\text { challenges [56]. }\end{array}$ \\
\hline & $\begin{array}{l}\text { 1.3. Scalability limitation of } \\
\text { the blockchain network [23] to } \\
\text { deal with the transactions } \\
\text { data variety, velocity and } \\
\text { volume }[15,23]\end{array}$ & $\mathrm{T}$ & $\begin{array}{c}\text { The scalability of private, consortium, and public } \\
\text { blockchains need to be all practically tested for the } \\
\text { transactions' throughput of BECOM projects. } \\
\text { Security and decentralization should be adequately } \\
\text { maintained [25]. }\end{array}$ \\
\hline
\end{tabular}


Table 3. Cont.

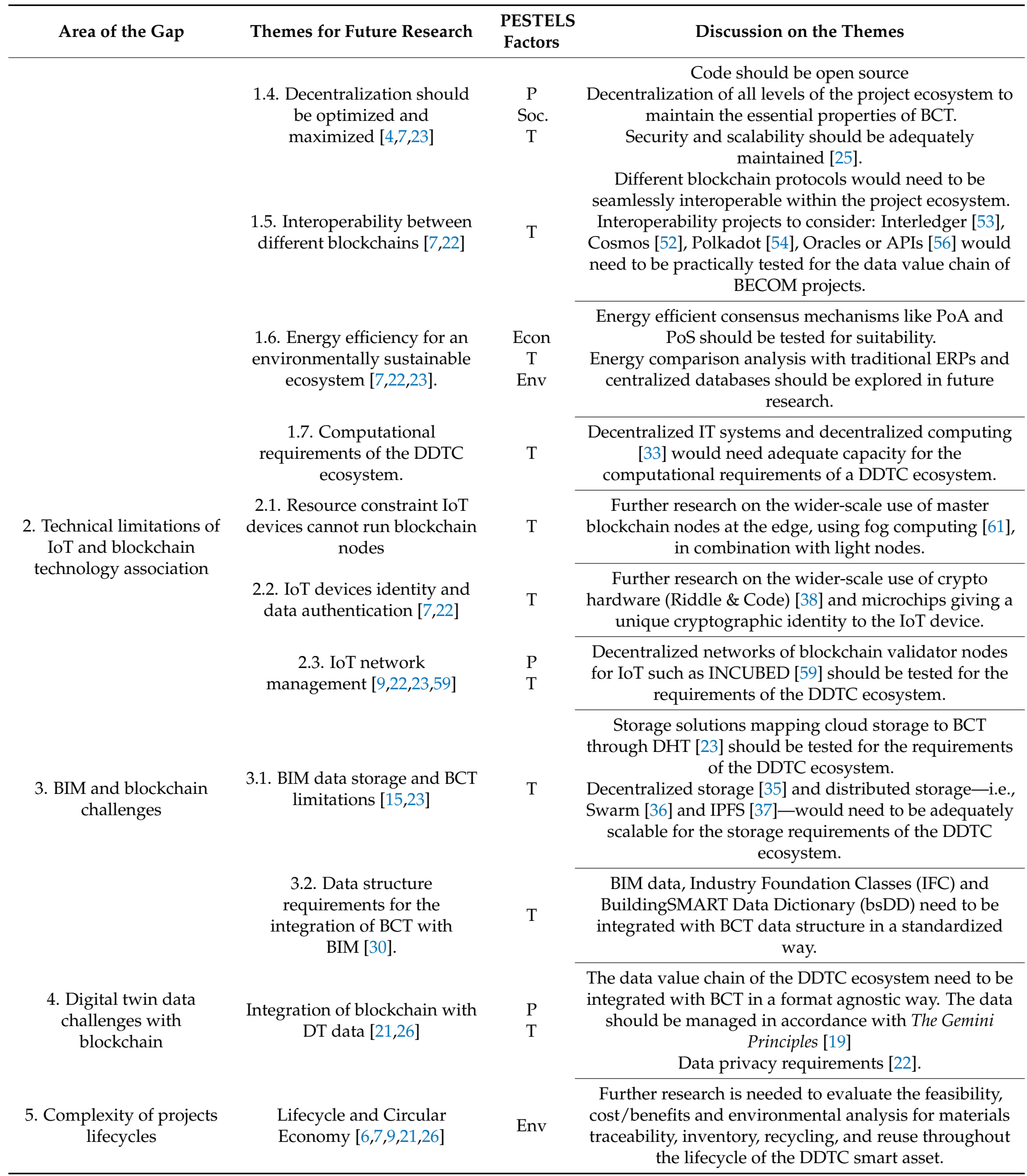

The suitability and limitations of the DDTC framework for the BECOM industry 4.0 can then be evaluated against these gaps; and future research directions are also identified in this section. 
The governance of decentralized blockchain networks is a key challenge. Unlike previous studies, the DDTC model proposes using a combination of the private, consortium, and public blockchain networks to narrow this gap. These three networks would then comprise the core elements of the back-end blockchain layer, as shown in Figure 2. These blockchain networks have different levels of governance (private, consortium, and public) adapted to the group level they respectively refer to (enterprise, project, or public) as discussed in Section 5. These blockchain networks should be interoperable with each other to enable connectivity within the ecosystem. Essential project data can be anchored or bridged from an enterprise-level private blockchain to a common project-based consortium blockchain facilitating transactions between project-wide stakeholders in a secure and interoperable manner. Private and consortium blockchains can use efficient consensus mechanisms such as proof-of-authority (PoA) [44], which is scalable, energy-efficient, and has minimal transaction fees. Additionally, the PoA consensus mechanism can ensure role-based access control, a key requirement for DT ecosystems [17]. However, the suitability of PoA consensus mechanisms for the functional and non-functional requirements of blockchain applications for the BECOM industry data value chain would need to be practically verified by further research works. Private and consortium blockchains networks data can be anchored into public blockchains when it is required to make key project information auditable by the public in order to improve trust, transparency, and integrity of the data. As shown on Figure 2, the blockchain layer of the DDTC model includes public blockchains as a key component. These public networks should have sufficient throughput; hence, platforms such as Ethereum 2.0 [45], Cardano [47], or Solana [65] could be explored for future experimentations. Indeed, these public blockchain platforms use proof-of-stake (PoS) (and proof-of-history ( $\mathrm{PoH}$ ) for Solana) consensus mechanisms, that are respectively called Casper [46] (for Ethereum 2.0) and Ouroboros [48] (for Cardano). These efficient consensus mechanisms are significantly more scalable and energy efficient than PoW mining. Moreover, these public blockchains integrate native cryptocurrencies [60] that enable incentivization for participants to 'play by the rules'. They also integrate smart contracts to automate processes such as payments, purchase orders, contracts, decentralize the funding of construction projects ( $\mathrm{Li}$ et al., 2019), and enable the tokenization of digital or physical assets. Alternatively, with less scalable blockchain such as Ethereum 1.0 [32], transactional data and states can be anchored on the blockchain by packet at regular time intervals. This method is viable to overcome scalability limitations for the Exonum and Stadjerspas projects [40]. Additionally, layer two "off-chain" scaling solutions like Plasma [66] or Raiden Network [67] can overcome the scalability limitations of public blockchain with low throughput such as Ethereum 1.0.

Regarding the blockchain interoperability challenges revealed in Table 3, the DDTC model proposes to leverage interoperability protocols such as Interledger [53], Cosmos [52], and Polkadot [54] to overcome interoperability issues. To integrate BCT with IoT, the DDTC aims to extend the decentralization of IoT management with protocols like INCUBED [59]. Additionally, IoT devices' identity and data authentication can be secured with crypto hardware [20] secure elements [38].

Data storage limitations of $\mathrm{BCT}$ form a major gap and key challenge making it impossible to process large volumes of data (from BIM, IoT, or DT) on the blockchain. To narrow this gap area, the DDTC model suggests as a first approach to leverage DHT to map resilient data storage systems with blockchain hash proofs [26]. However, this approach is limited because the actual data would remain off the blockchain, and only its digital fingerprint would be on the blockchain. Hence, decentralized database [24] and decentralized cloud storage [35] blockchain-based systems should be considered to enable the decentralization and security of data storage for DTs. Moreover, distributed storage systems like IPFS [37] and Swarm [36] that use DHT can also be used. However, these DHT based distributed storage platforms have limited storage capacity and do not facilitate data privacy. These limitations make them potentially inadequate for the Big Data volume and privacy requirements of the information value chain from projects of the BECOM industry. 
The integration of blockchain with cyber-physical systems (BCPS) like DT is another gap area since these technologies are emerging and the literature and practical implementations in this field are scarce. The DDTC model is a BCPS [22] with four layers that include a blockchain layer, a computing layer, a data management layer, and a connection layer. The back-end computing layer is composed of blockchain protocols (including open-source public blockchains), smart contracts, DApps, DAOs, DHT, oracles, interoperability protocols, decentralized cloud computing systems, distributed storage systems, access control mechanisms, edge and fog computing, and privacy layers as required. The role of DDTC connection layer is to capture project data from the 'real world' via IoT and oracles, and from software via APIs. These data are initially filtered in accordance with The Gemini Principles, and transferred from the connection layer to the data management layer. The DDTC data management layer ensures that information management also follows The Gemini Principles using, for example, data cleansing, data curation, data structures, data mining, data modeling, and data analytics techniques, so that the data volume decrease while the data value is increased [19]. Moreover, this decrease in data volume is beneficial for the storage limitation of BCT and contributes to filtering the data so that only valuable data are anchored on the blockchain. The data privacy requirements for BECOM project forms a gap when it comes to public or consortium blockchains. Indeed, consortium and public BCT are open to multiple stakeholders and data privacy and confidentiality of transactions can be a challenge. Moreover, the GDPR privacy right to erasure [28] is not well compatible with BCT since certain project data may not need to be retained after the project life cycle end. Hence, public blockchains would not be fit for the purpose of specific data requiring to be deleted at a certain point in time. Future research works should define the identification and categorization of project data requiring to be deleted or to remain permanently stored. The DDTC model aims to address the privacy requirement with the components of the private blockchain of the core blockchain layer. Indeed, the enterprises and stakeholders that require to keep certain data private would simply not transact them publicly and transact them only through their private blockchain networks. Alternatively, privacy protocols or encryptions mechanisms may be explored, as mentioned in Section 4.

The DDTC model is beneficial for BECOM projects as it can increase efficiency, productivity, transparency, trust, and security for the project data value chain. The DDTC is cyclic to integrate the $\mathrm{CE}$ and improve sustainability by reducing construction wastes and facilitating reuse and recycling through the traceability of materials enabled by BCT [64] throughout project lifecycles. The DDTC also provides a transparent (or private as needed), traceable, and secured unified information management framework that can address the complexity of data management at all phases of BECOM projects and contribute to reducing data silos in the industry. Furthermore, the DDTC model can integrate decentralized finance (DeFi) protocols [68] by leveraging smart contracts and allowing projects participants and the public to exchange value securely in a trustworthy and peer-to-peer way, with the financial ecosystems of smart assets represented by their DTs. The combination of DeFi with the CE can lead to the formation of a decentralized CE (DeCE) which could be the object of future research works. In a fully decentralized context, the DDTC model could run along with DAOs [9] to automate organizations' processes in the back-end of DT applications. Peer-to-peer blockchain networks can also facilitate decentralized collaborations between key project stakeholders such as clients, engineers, architects, contractors, regulators, facility managers, and owners. Moreover, BCT would secure the sharing of information within DCDEs that record historical data throughout the lifecycle of projects. Common data environments (CDE) could be "distributed across different computers" [63], acting as blockchain nodes and recording information historical sates changes within the CDE [69], and hence forming a DCDE [9]. Such a distributed collaborative environment could span from the project information model (PIM) to the asset information model (AIM) and cover the full lifecycle of smart infrastructure assets. Big Data from the project information value chain would be managed in accordance with The Gemini Principles [19] before they are stored on DCDEs. The DDTC model also considers strengthening the implementa- 
tion of reforms and regulations within the industry through the integration of regulatory blockchain nodes validating key transactions throughout the lifecycle of projects.

This study provides the novel DDTC framework, its technological components, and the key technological gaps identified. Hence the theoretical implications of this study are for industry practitioners to develop the theoretical framework further, standardize it, and contribute to closing the gaps. The DDTC provides the key technological building blocks for the adoption of BCT for DT in order to improve some key challenges of the BECOM industry. Hence the practical implications of this study will contribute to enhancing the BECOM industry 4.0 with the DDTC model that aims to facilitate trusted collaborations, improve information sharing, enhance information management [7] and security, and promote environmental sustainability. The DDTC model promotes the integrity of the data value chain and can guarantee the trustworthiness of the information that is handed over at each phase of the project lifecycle. The DDTC model is decentralized and contributes to a paradigm shift towards a democratization of the BECOM industry by redistributing control to individuals [7] through the decentralization of services and the disintermediation of centralized third parties. This decentralized model leverages incentivized data sharing in a peer-to-peer way to reduce the adversarial behaviors in the BECOM industry that are limiting collaboration and information sharing. This paradigm shift also leverages automation of processes with smart contracts to improve costs savings, efficiency [7], and productivity. The DDTC model improves safety [2], transparency, cybersecurity, information integrity, openness, and environmental sustainability for the BECOM industry. Finally, the DDTC is beneficial for the industry and the public good; and it creates value, provides smart insights, and is aligned with The Gemini Principles [19].

\section{Conclusions}

The paper has developed a framework for the integration of BCT with DT for BECOM projects in the context of industry 4.0. The three objectives were to identify the challenges of the BECOM industry 4.0 affecting BCT adoption, evaluate the key technological factors affecting the adoption of $\mathrm{BCT}$, and propose a conceptual model facilitating the integration of $\mathrm{BCT}$ and DT for projects of the BECOM industry 4.0. The originality of this paper comprises the proposed technological framework based on a novel conceptual model called the decentralized digital twin cycle (DDTC). This model promotes ecosystems of trusted, decentralized, and sustainable DTs for the BECOM industry 4.0 where BCT secures information sharing for the data value chain of projects. Moreover, the DDTC model contributes to narrowing the technological gaps identified about BCT adoption for DT for industry 4.0.

The key challenges, affecting BCT adoption in the industry, that were identified from the PESTELS review of the literature are the lack of trust, lack of transparency, lack of traceability of information, inefficiencies, blockchain networks governance, lack of collaboration, fragmentation in data silos, high implementation costs, lack of productivity, adversarial practices, poor digitization, cybersecurity risks, difficulties enforcing standards and regulations, lack of accountability, data interoperability, blockchain interoperability, and environmental sustainability. The key technological factors affecting BCT adoption in the BECOM industry 4.0 relate to the decentralization of systems and networks, stringent requirements for Big Data, specific IT requirements for BCT, integration of smart contracts, identity management and access control, blockchain properties and performances, and the integration of BCT with the key technologies of the BECOM industry 4.0: BIM, IoT, and DT.

Another originality of the paper is the identification of technological gaps affecting BCT adoption for DT in the BECOM industry 4.0. The gaps areas identified in the literature are: the technical and non-functional requirements for $\mathrm{BCT}$ applications in the BECOM industry 4.0, and the technological challenges related to the integration of BCT with BIM, IoT, and DT throughout complex projects' lifecycles.

The DDTC model leverages BCT to strengthen the data value chain in terms of data sharing, cybersecurity, data integrity, immutability, traceability, and transparency or 
privacy of information. The DDTC model can reduce data silos in the BECOM industry by leveraging decentralization of IT infrastructures. This decentralization is achieved with key technological components, such as DCDEs, to enhance collaboration and information sharing, smart contracts, DApps, DAOs, decentralized storage systems, decentralized cloud computing systems, decentralized management of IoT networks, decentralized oracles, and decentralized governance for blockchain networks. Project stakeholders (consultants, engineers, architects, contractors, regulators, facility managers, owners, and users) can share transactional data in a peer-to-peer way and validate transactions on the blockchain shared ledger to ensure data integrity. The security and integrity of IoT data are enhanced with crypto hardware to cryptographically secure IoT devices' identities and ensure the authentication of IoT data. Thus, the DDTC framework ensures that the sensors' data input is secure and trustworthy. To overcome data storage limitations of $\mathrm{BCT}$, the Big Data volumes involved throughout BECOM projects can be stored in decentralized cloud storage and mapped to the blockchain through DHT. The model considers interblockchain solutions to address interoperability challenges. Smart contracts are leveraged for automation of processes in order to reduce time and costs and improve efficiency and productivity in the BECOM industry 4.0. Efficient consensus mechanisms (e.g., PoA and $\mathrm{PoS}$ ) are proposed for adequate scalability of the blockchain layers that require high throughput and low latency for high data velocity. Moreover, such consensus mechanisms are energy efficient to minimize the project's carbon footprint. Additionally, the DDTC model is cyclic to promote sustainability and minimize waste by reusing and recycling materials for the CE and the DeCE. Hence, the DDTC technological framework proposed addresses some key industry challenges and technological factors, and it contributes to narrowing the gaps identified in the literature.

This paper is limited to examining the current literature and state of the art on blockchain technology and its applications for DT in the BECOM industry 4.0. Future studies are suggested including practical experiments for implementing the proposed framework requiring to be developed and tested. The future studies which are experimentation based can reveal and validate that sufficient scalability, energy efficiency, and cost savings can be achieved with blockchain protocols adequately chosen for DTs of the BECOM industry 4.0. Moreover, the blockchain consensus mechanisms discussed in this paper should be tested with high data volume, velocity, and variety that simulate the real conditions of the data value chain from a project of the BECOM industry 4.0. Thus, future works should test if BCT can perform adequately in terms of scalability, energy efficiency, cost (infrastructure deployment cost and transaction fees), as well as the integration of $\mathrm{BCT}$ with other key technologies like BIM, IoT, and DT. Additionally, future research studies should work on the classification of project information to identify the data categories (from the BECOM projects information value chain) that require the most to be anchored in the blockchain layer (private, consortium, or public) of the DDTC. Future work should also identify the categories of project data that require security, transparency, privacy, openness, immutability, deletion, or anchorage into the blockchain for each phase of the project lifecycle. Furthermore, future research is required to develop open data standards to standardize key project data schemas and enable a structured data layer to integrate project data, BIM data, IoT data, DT data, and asset information into the blockchain. Additionally, the standardization for the project information value chain would require compliance with The Gemini Principles. Finally, future works should investigate the decentralization of collaborative financial ecosystems in the BECOM industry 4.0 and its integration to the $\mathrm{CE}$ to form a DeCE.

Author Contributions: Conceptualization, B.T.; methodology, B.T. and S.S.; validation, B.T. and S.S.; formal analysis, B.T.; investigation, B.T.; resources, B.T.; data curation, B.T.; writing—original draft preparation, B.T.; writing—review and editing, B.T. and S.S.; visualization, B.T.; supervision, S.S. All authors have read and agreed to the published version of the manuscript.

Funding: This research received no external funding. 
Institutional Review Board Statement: Not applicable.

Informed Consent Statement: Not applicable.

Data Availability Statement: The data used is from the literature presented in this paper.

Acknowledgments: The authors of this paper acknowledge the support of the Australian Government for this research.

Conflicts of Interest: The authors declare no conflict of interest.

\section{Abbreviations}

$\begin{array}{ll}\text { AEC } & \text { Architecture, engineering and construction } \\ \text { AIM } & \text { Asset information model } \\ \text { API } & \text { Application programming interface } \\ \text { BCT } & \text { Blockchain technology } \\ \text { BCPS } & \text { Blockchain with cyber-physical systems } \\ \text { BECOM } & \text { Building, engineering, construction, operations, and mining } \\ \text { BIM } & \text { Building information modelling } \\ \text { bsDD } & \text { BuildingSMART data dictionary } \\ \text { CDE } & \text { Common data environment } \\ \text { CE } & \text { Circular economy } \\ \text { DCDE } & \text { Decentralized common data environment } \\ \text { DeCE } & \text { Decentralized circular economy } \\ \text { DDTC } & \text { Decentralized digital twin cycle } \\ \text { CAD } & \text { Computer-aided design } \\ \text { COBie } & \text { Construction operations building information exchange } \\ \text { CPS } & \text { Cyber physical system } \\ \text { DAO } & \text { Decentralized autonomous organizations } \\ \text { DApp } & \text { Decentralized application } \\ \text { DeFi } & \text { Decentralized finance } \\ \text { DHT } & \text { Distributed hash table } \\ \text { DT } & \text { Digital twin } \\ \text { FM } & \text { Facility management } \\ \text { GDPR } & \text { General data protection regulation } \\ \text { GIGO } & \text { Garbage in garbage out } \\ \text { GIS } & \text { Geographic information system } \\ \text { IFC } & \text { Industry foundation classes } \\ \text { IoT } & \text { Internet of things } \\ \text { IPFS } & \text { The interplanetary file system } \\ \text { IT } & \text { Information technology } \\ \text { PoA } & \text { Proof of authority } \\ \text { PoS } & \text { Proof of stake } \\ \text { PoW } & \text { Proof of work } \\ \text { PESTELS } & \text { Political, economic, social, technological, environmental, legal, and security } \\ \text { PIM } & \text { Project information model } \\ \text { RFID } & \text { Radio-frequency identification } \\ \text { UI } & \text { User interface } \\ \text { XML } & \text { Extensible markup language } \\ & \end{array}$

\section{References}

1. Oesterreich, T.D.; Teuteberg, F. Understanding the implications of digitisation and automation in the context of Industry 4.0: A triangulation approach and elements of a research agenda for the construction industry. Comput. Ind. 2016, 83, 121-139. [CrossRef]

2. Alaloul, W.S.; Liew, M.; Zawawi, N.A.W.A.; Kennedy, I.B. Industrial Revolution 4.0 in the construction industry: Challenges and opportunities for stakeholders. Ain Shams Eng. J. 2019, 11, 225-230. [CrossRef]

3. Nawari, N.O.; Ravindran, S. Blockchain technology and BIM process: Review and potential applications. J. Inf. Technol. Constr. (ITcon) 2019, 24, 209-238. 
4. Mason, J. BIM fork: Are smart contracts in construction more likely to prosper with or without BIM? J. Leg. Aff. Disput. Resolut. Eng. Constr. 2019, 11, 02519002. [CrossRef]

5. IBM. Engineering and Construction Digital Supply Chain; IBM: Amonk, NY, USA, 2019.

6. Wang, Z.; Wang, T.; Hu, H.; Gong, J.; Ren, X.; Xiao, Q. Blockchain-based framework for improving supply chain traceability and information sharing in precast construction. Autom. Constr. 2020, 111, 103063. [CrossRef]

7. Li, J.; Greenwood, D.; Kassem, M. Blockchain in the built environment and construction industry: A systematic review, conceptual models and practical use cases. Autom. Constr. 2019, 102, 288-307. [CrossRef]

8. McKinsey. The Internet of Things: Mapping the Value Beyond the Hype; McKinsey \& Company: New York, NY, USA, 2015.

9. Ye, Z.; Yin, M.; Tang, L.; Jiang, H. Cup-of-Water theory: A review on the interaction of BIM, IoT and blockchain during the whole building lifecycle. in ISARC. In Proceedings of the International Symposium on Automation and Robotics in Construction, Berlin, Germany, 20-25 July 2018.

10. McNamara, A.J.; Sepasgozar, S.M. Developing a theoretical framework for intelligent contract acceptance. Constr. Innov. 2020, 20, 421-445. [CrossRef]

11. Nakamoto, S. Bitcoin: A Peer-to-Peer Electronic Cash System. 2008. Available online: https://bitcoin.org/bitcoin.pdf (accessed on 23 October 2019).

12. Australian Government. The National Blockchain Roadmap: Progressing towards a Blockchain-Empowered Future. 2020. Available online: https: / /www.industry.gov.au/sites/default/ files/2020-02/national-blockchain-roadmap.pdf (accessed on 3 March 2020).

13. Arup, Blockchain Technology, How the Inventions Behind Bitcoin are Enabling a Network of Trust for the Built Environment. 2017. Available online: https://www.arup.com/perspectives/publications/research/section/blockchain-technology (accessed on 18 May 2020).

14. Shirowzhan, S.; Tan, W.; Sepasgozar, S.M. Digital Twin and CyberGIS for Improving Connectivity and Measuring the Impact of Infrastructure Construction Planning in Smart Cities; Multidisciplinary Digital Publishing Institute: Basel, Switzerland, 2020.

15. Turk, Ž.; Klinc, R. Potentials of blockchain technology for construction management. Procedia Eng. 2017, 196, 638-645. [CrossRef]

16. Shergold, P.; Weir, B. Building Confidence: Improving the Effectiveness of Compliance and Enforcement Systems for the Building and Construction Industry across Australia. Aust. Inst. Build. Surv. 2018. Available online: https://www.industry.gov.au/sites/ default/files/July\%202018/document/pdf/building_ministers_forum_expert_assessment_-_building_confidence.pdf (accessed on 12 December 2021).

17. ANZLIC. Principles for Spatially Enabled Digital Twins of the Built and Natural Environment in Australia; ANZLIC: Barton, Australia, 2019. Available online: https:/ / www.anzlic.gov.au/sites / default/files/files/principles_for_spatially_enabled_digital_twins_ of_the_built_and_natural_.pdf (accessed on 12 December 2021).

18. Mott MacDonald. Digital Twins, Better Outcomes from Connected Data; Mott MacDonald: Croydon, UK, 2019.

19. CDBB. Gemini Principles; Centre for Digital Built Britain. 2018. Available online: https://www.cdbb.cam.ac.uk/system/files/ documents/TheGeminiPrinciples.pdf (accessed on 18 May 2020).

20. Sallaba, M.; Siegel, D.; Becker, S. IoT Powered by Blockchain-How Blockchains Facilitate the Application of Digital Twins in IoT. Deloitte Issue 2018. Available online: https: / www2.deloitte.com/content/dam/Deloitte/de/Documents/Innovation/IoTpowered-by-Blockchain-Deloitte.pdf (accessed on 27 December 2019).

21. Mandolla, C.; Petruzzelli, A.M.; Percoco, G.; Urbinati, A. Building a digital twin for additive manufacturing through the exploitation of blockchain: A case analysis of the aircraft industry. Comput. Ind. 2019, 109, 134-152. [CrossRef]

22. Lee, J.; Azamfar, M.; Singh, J. A blockchain enabled Cyber-Physical System architecture for Industry 4.0 manufacturing systems. Manuf. Lett. 2019, 20, 34-39. [CrossRef]

23. Panarello, A.; Tapas, N.; Merlino, G.; Longo, F.; Puliafito, A. Blockchain and IoT integration: A systematic survey. Sensors 2018, 18, 2575. [CrossRef]

24. Bigchain, D.B.; GmbH, B. BigchainDB 2.0 The Blockchain Database. Germany. 2018. Available online: https://www.bigchaindb. com/whitepaper/bigchaindb-whitepaper.pdf (accessed on 23 October 2019).

25. Bez, M.; Fornari, G.; Vardanega, T. The scalability challenge of ethereum: An initial quantitative analysis. In Proceedings of the 2019 IEEE International Conference on Service-Oriented System Engineering (SOSE), San Francisco East Bay, CA, USA, 4-9 April 2019.

26. Dietz, M.; Putz, B.; Pernul, G. A Distributed Ledger Approach to Digital Twin Secure Data Sharing. In Proceedings of the IFIP Annual Conference on Data and Applications Security and Privacy, Calgary, AB, Canada, 25-26 June 2019; Springer: Berlin/Heidelberg, Germany, 2019.

27. Ellen Macarthur Foundation. Cities and the Circular Economy. Available online: https://www.ellenmacarthurfoundation.org/ explore/ cities-and-the-circular-economy (accessed on 12 December 2021).

28. Finck, M. Blockchain and the General Data Protection Regulation; European Parliament: Strasbourg, France, 2019.

29. Li, Z.; Yang, Z.; Xie, S. Computing Resource Trading for Edge-Cloud-assisted Internet of Things. IEEE Trans. Ind. Inform. 2019, 15, 3661-3669. [CrossRef]

30. Zheng, R.; Jiang, J.; Hao, X.; Ren, W.; Xiong, F.; Ren, Y. bcBIM: A Blockchain-Based Big Data Model for BIM Modification Audit and Provenance in Mobile Cloud. Available online: https:/ / www.hindawi.com/journals/mpe/2019/5349538/ (accessed on 19 January 2020). 
31. Kieselmann, O.; Wacker, A.; Schiele, G. k-rAC: A Fine-Grained k-Resilient Access Control Scheme for Distributed Hash Tables. In Proceedings of the 12th International Conference on Availability, Reliability and Security, Reggio Calabria, Italy, 29 August1 September 2017.

32. Buterin, V. Ethereum Whitepaper. 2013. Available online: https:/ / ethereum.org/en/whitepaper/ (accessed on 15 June 2020$).$

33. Fedak, G.; Wassim, B.; Eduardo, A. iExec: Blockchain-Based Decentralized Cloud Computing. 2018. Available online: https:/ /iex.ec/wp-content/uploads/pdf/iExec-WPv3.0-English.pdf (accessed on 3 November 2019).

34. Hanke, T.; Movahedi, M.; Williams, D. Dfinity technology overview series, consensus system. arXiv 2018, arXiv:1805.04548.

35. Storj Labs, I. Storj: A Decentralized Cloud Storage Network Framework. 2018. Available online: https://www.storj.io/storj.pdf (accessed on 5 November 2011).

36. Trón, V. The Book of Swarm. 17 November 2020, 2020 Release 1.0. Available online: https://www.ethswarm.org/The-Book-ofSwarm.pdf (accessed on 17 December 2021).

37. Benet, J. Ipfs-content addressed, versioned, p2p file system. arXiv 2014, arXiv:1407.3561.

38. Riddle \& Code. The Blockchain Technology Company. Available online: https://www.riddleandcode.com/ (accessed on 17 May 2021).

39. Ellis, S.; Juels, A.; Nazarov, S. Chainlink: A decentralized oracle network. Retrieved March 2017, 11, 2018.

40. Allessie, D.; Sobolewski, M.; Vaccari, L. Blockchain for Digital Government: An Assessment of Pioneering Implementations in Public services; Joint Research Centre (Seville Site): Sevilla, Spain, 2019.

41. Fabian Vogelsteller. ERC-725 Ethereum Identity Standard. Available online: https://erc725alliance.org (accessed on 12 December 2021).

42. Stokkink, Q.; Pouwelse, J. Deployment of a blockchain-based self-sovereign identity. In Proceedings of the 2018 IEEE International Conference on Internet of Things (iThings) and IEEE Green Computing and Communications (GreenCom) and IEEE Cyber, Physical and Social Computing (CPSCom) and IEEE Smart Data (SmartData), Halifax, NS, Canada, 20-23 August 2018; pp. 1336-1342.

43. Prada-Delgado, M.Á.; Baturone, I.; Dittmann, G.; Jelitto, J.; Kind, A. PUF-derived IoT identities in a zero-knowledge protocol for blockchain. Internet Things 2020, 9, 100057. [CrossRef]

44. Singh, P.K.; Singh, R.; Nandi, S.K.; Nandi, S. Managing smart home appliances with proof of authority and blockchain. In Proceedings of the International Conference on Innovations for Community Services, Bhubaneswar, India, 12-14 January 2019; Springer: Berlin/Heidelberg, Germany, 2019.

45. Ethereum. Ethereum 2.0 Specifications. Available online: https://github.com/ethereum/eth2.0-specs (accessed on 12 December 2021).

46. Buterin, V.; Griffith, V. Casper the friendly finality gadget. arXiv 2017, arXiv:1710.09437.

47. IOHK. Cardano. Available online: https://www.cardano.org/ (accessed on 12 December 2021).

48. David, B.M.; Gazi, P.; Kiayias, A.; Russell, A. Ouroboros Praos: An adaptively-secure, semi-synchronous proof-of-stake protocol. IACR Cryptol. ePrint Arch. 2017, 2017, 573.

49. Williamson, Z.J. The AZTEC Protocol; 2018. Version 1.0.1. Available online: https://github.com/AztecProtocol/AZTEC/blob/ (accessed on 21 December 2019).

50. Zyskind, G.; Nathan, O.; Pentland, A. Enigma: Decentralized computation platform with guaranteed privacy. arXiv 2015, arXiv:1506.03471.

51. OASIS. Baseline Protocol. 2020. Available online: https:/ / docs.baseline-protocol.org/ (accessed on 12 December 2021).

52. Kwon, J.; Buchman, E. Cosmos Whitepaper. A Netw. Distrib. Ledgers 2019. Available online: https://v1.cosmos.network/ resources/whitepaper (accessed on 10 November 2019).

53. Thomas, S.; Schwartz, E. A Protocol for Interledger Payments. 2015. Available online: https://interledger.org/interledger.pdf (accessed on 12 December 2021).

54. Wood, G. Polkadot: Vision for a Heterogeneous Multi-Chain Framework; White Paper; 2016. Available online: https://polkadot. network/PolkaDotPaper.pdf (accessed on 10 November 2019).

55. Verdian, G.; Tasca, P.; Paterson, C.; Mondelli, G. Quant Overledger Whitepaper; 2018. Available online: https://girishmehta.com/ projects / quant/wp-content/uploads/2020/07/Quant_Overledger_Whitepaper-Sep-1.pdf (accessed on 24 May 2020).

56. World Economic Forum. Inclusive Deployment of Blockchain for Supply Chains: Part 6-A framework for Blockchain Interoperability; 2020; Part 6. Available online: https://www3.weforum.org/docs/WEF_A_Framework_for_Blockchain_Interoperability_2020.pdf (accessed on 18 May 2020).

57. BIMCHAIN. Accelerating BIM through the Blockchain. Delivering the Trust in the Data BIM Needs. Available online: https:/ / ascelibrary.org/doi/10.1061/9780784482865.124 (accessed on 5 May 2020).

58. Bosurgi, G.; Celauro, C.; Pellegrino, O.; Rustica, N.; Giuseppe, S. The BIM (Building Information Modeling)-Based Approach for Road Pavement Maintenance. In International Symposium on Asphalt Pavement E Environment. Available online: https:/ / link.springer.com/chapter/10.1007/978-3-030-29779-4_47 (accessed on 20 January 2020).

59. Slock.it. Connects Devices to the Blockchain, Enabling the Economy of Things. Available online: https://slock.it/ (accessed on 8 March 2020).

60. Pustišek, M.; Kos, A. Approaches to front-end iot application development for the ethereum blockchain. Procedia Comput. Sci. 2018, 129, 410-419. [CrossRef] 
61. Nyamtiga, B.W.; Sicato, J.C.S.; Rathore, S.; Sung, Y.; Park, J.H. Blockchain-Based Secure Storage Management with Edge Computing for IoT. Electronics 2019, 8, 828. [CrossRef]

62. Revibe Energy. Vibration Energy Harvesting. Available online: https://revibeenergy.com/energy-harvesting/ (accessed on 24 May 2020).

63. ISO 19650-1: 2018. Organization and Digitization of Information about Buildings and Civil Engineering Works, Including Building Information Modelling (BIM)_Information Management Using Building Information Modelling_Part 1: Concepts and Principles; International Organization for Standardization: Geneva, Switzerland, 2018.

64. Kouhizadeh, M.; Sarkis, J.; Zhu, Q. At the Nexus of Blockchain Technology, the Circular Economy, and Product Deletion. Appl. Sci. 2019, 9, 1712. [CrossRef]

65. Yakovenko, A. Solana: A New Architecture for a High Performance Blockchain v0. 8.13; Whitepaper. 2018. Available online: https:/ / solana.com/solana-whitepaper.pdf (accessed on 7 December 2021).

66. Poon, J.; Buterin, V. Plasma: Scalable autonomous smart contracts. White Paper 2017, 1-47.

67. Raiden Network. Fast, Cheap, Scalable Token Transfers for Ethereum. Available online: https://github.com/raiden-network/ raiden (accessed on 27 December 2019).

68. Chen, Y.; Bellavitis, C. Blockchain disruption and decentralized finance: The rise of decentralized business models. J. Bus. Ventur. Insights 2020, 13, e00151. [CrossRef]

69. ISO 19650-2: 2018. Organization and Digitization of Information about Buildings and Civil Engineering Works, Including Building Information Modelling (BIM)_Information Management Using Building Information Modelling_Part 2: Delivery Phase of the Assets; International Organization for Standardization: Geneva, Switzerland, 2018. 